\title{
Mutually Consistent Revealed Preference Demand Predictions
}

\author{
Abi Adams*
}

March 11, 2019

\begin{abstract}
Revealed preference restrictions are increasingly used to predict demand behaviour at new budgets of interest and as shape restrictions in nonparametric estimation exercises. However, the restrictions imposed are not sufficient for rationality when predictions are made at multiple budgets. I highlight the nonconvexities in the set of predictions that arise when making multiple predictions. I develop a mixed integer programming characterisation of the problem that can be used to impose rationality on multiple predictions. The approach is applied to the UK Family Expenditure Survey to recover rational demand predictions with substantially reduced computational resources compared to known alternatives.
\end{abstract}

\section{Introduction}

The revealed preference approach to demand prediction uses the behavioural hypothesis of utility maximisation in conjunction with a finite set of observations on a consumer's past behaviour to set identify demand responses at new budgets of interest. The benefits of such an approach are well understood: bounds can be placed on behavioural responses and welfare effects without the need for restrictive assumptions on consumer preferences. As Blundell (2005) argues, it is possible "to accomplish all that is required from parametric models of consumer behaviour using only nonparametric regression and revealed preference theory"; crucially, however, without placing strong restrictions on income and price responses.

${ }^{*}$ University of Oxford and Institute for Fiscal Studies. Email: abi.adams@economics.ox.ac.uk. I gratefully acknowledge funding from the Economic and Social Research Council (ESRC) under grant ES/I024808. I would like to thank Richard Blundell, Laurens Cherchye, Ian Crawford, Bram De Rock, Thomas Demuynck, Jeremias Prassl, and my four referees for helpful comments. I would like to thank Yuichi Kitamura and Jörg Stoye for the replication code underlying Kitamura and Stoye (2017). 
Since Varian (1982), revealed preference arguments have been successfully applied to predict consumer behaviour in a number of different scenarios including bounding demand responses for gasoline (Blundell, Kristensen, and Matzkin 2014), food (Blundell, Browning, and Crawford 2008), broadband (Varian 2012) and leisure (Manski 2014; Kline and Tartari 2016) among others. Recent methodological advances have, furthermore, the potential to extend the informativeness and scope of the approach. Nonparametric regression and rationality restrictions are increasingly combined to refine the set of revealed preference demand predictions (Blundell, Browning, and Crawford 2003; Blundell, Browning, and Crawford 2008; Blundell, Kristensen, and Matzkin 2014; Blundell, Browning, Cherchye, Crawford, De Rock, and Vermeulen 2015) or to recover theory-consistent, well-disciplined point estimates (Horowitz and Lee 2017; Blundell, Horowitz, and Parey 2017). The toolkit has also been developed to account for (nonadditive) heterogeneity in consumer preferences, moving the literature beyond deterministic choice models to allow for bounds to be placed on features of the distribution of demand (Blundell, Kristensen, and Matzkin 2014; Hoderlein and Stoye 2015; Kitamura and Stoye 2017).

This paper is concerned with whether the revealed preference restrictions imposed in the literature are sufficient for a set of predictions to be rational. It will be shown that, in general, when predictions are made at a set of budgets, they are not. In this context, application of the present restrictions (as set out in Varian (1982)) does not guarantee that the resulting set of predictions are jointly rational. The aim of this paper is to develop a practical characterisation of the set of mutually consistent demand predictions that can be implemented using reasonable computational resources by applied researchers.

This is of interest because applied problems often require demand behaviour to be forecast at multiple budgets. For example, researchers may be interested in comparing behavioural responses across a number of different policy reforms simultaneously. Furthermore, when revealed preference inequalities are used as shape restrictions to constrain nonparametric regression models, demand predictions are made over a grid of budget parameters. ${ }^{1}$ Indeed, the difficulty of imposing rationality on sets of nonparametric demand estimates has held back the practical application of recent methodological advances that combine revealed preference

\footnotetext{
${ }^{1}$ While theory consistency constraints may be imposed for a number of reasons beyond ensuring rationality of predictions (for example, decreasing the variance of nonparametric estimates and improving out-of-sample extrapolation properties - see Matzkin (1994) for a review), rationality is an important property for demand predictions if they are to be used for welfare analysis.
} 
restrictions with nonparametric regression. For example, if estimated income expansion paths violate rationality constraints, then they cannot be used to refine sets of revealed preference demand predictions as suggested in Blundell, Browning, and Crawford (2008). Note that estimated demands can violate revealed preference rationality restrictions simply due to sampling variation rather than any irrationality on the part of individual consumers or model misspecification.

In this paper, the revealed preference methodology is extended to address the prediction of rational demands over a set of new budgets. Revealed preference bounds do not extend without modification to scenarios in which multiple demand predictions are to be made because predictions across different budgets must be consistent with one another for them jointly to satisfy rationality restrictions - that is, rationality restrictions must hold between predictions (Blundell, Kristensen, and Matzkin 2014). This requirement of 'mutual consistency' of predictions generates nonlinearities in the standard revealed preference-type inequalities and results in a non-convex set of demand predictions, generating significant computation difficulties. ${ }^{2}$

It is shown that the revealed preference restrictions associated with our problem can be characterised as a mixed integer linear programme (MILP), which can be implemented with reasonable computational resources. Approaches for enhancing the efficiency of the programming problem are put forward and connections are made to the recent methodological advances of Hoderlein and Stoye (2015) and Kitamura and Stoye (2017). The practical use of the methodology is demonstrated via an illustrative application to consumer microdata from the UK Family Expenditure Survey. We show that the approach put forward in this paper can generate significant computational savings over known alternatives (e.g. modification of the rational types methodology developed in Kitamura and Stoye (2017)) and allows one to recover theory-consistent nonparametric demand estimates with ease, permitting straightforward application of the techniques introduced by Blundell, Browning, and Crawford (2008).

This paper proceeds as follows. Section 2 gives an overview of the revealed preference approach to demand prediction, including recent methodological advances that make use of nonparametric income expansion path estimates. Section 3 highlights the need for mutual consistency of demand predictions, links this problem to difficulties in imposing rationality on nonparametric demand systems more broadly, and discusses the

\footnotetext{
${ }^{2}$ See Section 4 of Blundell, Kristensen, and Matzkin (2014) for a discussion of this point and their proposed two-step estimation strategy to estimate rational income expansion paths.
} 
strategies currently available for generating jointly rational demands. Section 4 develops a practical mixed integer programming characterisation of mutually consistent rationality constraints. Section 5 demonstrates the practical relevance of the characterisation, showing that it can be applied in contexts often encountered in applied demand analysis with reasonable computational resources. Finally, Section 6 concludes. Proofs of all propositions are in the Appendix.

\section{Revealed Preference Restrictions in Demand Analysis}

Varian (1982) first demonstrated how revealed preference arguments can be used to predict counterfactual demands. To illustrate, imagine that we are interested in a consumer's demand behaviour at a finite number of budgets $\left\{\mathbf{p}_{i}, x_{i}\right\}_{i=1, \ldots, B}$, where $\mathbf{p}_{i} \in \mathbb{R}_{++}^{K}$ and $x_{i}>0$ give prices and total expenditure at budget $i$ respectively. Consumer behaviour is observed at a subset of these budgets, $\mathscr{D}=\{1, \ldots, T\}$, where $T<B$. We wish to use the panel of observations $\left\{\mathbf{p}_{t}, \mathbf{q}_{t}\right\}_{t=1, \ldots, T}$ to predict behaviour at the remaining subset of budgets, $\mathscr{P}=\{T+1, \ldots, B\}$.

Throughout this paper, I assume that the choice behaviour of consumers is rational, where rationality is equated with choice behaviour that satisfies the Generalised Axiom of Revealed Preference (GARP), defined below. ${ }^{3}$ GARP is a consistency condition. Intuitively, if an individual 'reveals a preference' for some bundle $\mathbf{q}_{t}$ over another bundle $\mathbf{q}_{s}$ by selecting $\mathbf{q}_{t}$ when $\mathbf{q}_{s}$ is available, then that individual cannot choose $\mathbf{q}_{s}$ over $\mathbf{q}_{t}$ in an alternative choice scenario in which $\mathbf{q}_{t}$ is available.

Direct revealed preference. If $\mathbf{p}_{t}^{\prime} \mathbf{q}_{s} \leq x_{t}$, then $\mathbf{q}_{t}$ is directly revealed preferred to $\mathbf{q}_{s}$, or $\mathbf{q}_{t} \mathbb{R}^{0} \mathbf{q}_{s}$. Further, the budget $\left\{\mathbf{p}_{t}, x_{t}\right\}$ is indirectly direct revealed preferred to the budget $\left\{\mathbf{p}_{s}, x_{s}\right\}$, or $\left\{\mathbf{p}_{t}, x_{t}\right\} \mathbb{R}^{0}\left\{\mathbf{p}_{s}, x_{s}\right\}$.

Direct strict revealed preference. If $\mathbf{p}_{t}^{\prime} \mathbf{q}_{s}<x_{t}$, then $\mathbf{q}_{t}$ is directly strictly revealed preferred to $\mathbf{q}_{s}$, or $\mathbf{q}_{t} \mathbb{P}^{0} \mathbf{q}_{s}$. Further, the budget $\left\{\mathbf{p}_{t}, x_{t}\right\}$ is indirectly directly strictly revealed preferred to $\left\{\mathbf{p}_{s}, x_{s}\right\}$, or $\left\{\mathbf{p}_{t}, x_{t}\right\} \mathbb{P}^{0}\left\{\mathbf{p}_{s}, x_{s}\right\}$

\footnotetext{
${ }^{3}$ See Apesteguia and Ballester (2015), Dean and Martin (2016), Echenique, Lee, and Shum (2011) and Varian (1990) for approaches to measure violations of rationality.
} 
Revealed preference. If $\mathbf{q}_{t} \mathbb{R}^{0} \mathbf{q}_{u}, \mathbf{q}_{u} \mathbb{R}^{0} \mathbf{q}_{v}, \ldots, \mathbf{q}_{w} \mathbb{R}^{0} \mathbf{q}_{s}$, then $\mathbf{q}_{t}$ is revealed preferred to $\mathbf{q}_{s}$, $\mathbf{q}_{t} \mathbb{R} \mathbf{q}_{s}$. The analogous definition holds for the indirect revealed preference relation.

Strict revealed preference. If $\mathbf{q}_{t} \mathbb{P}^{0} \mathbf{q}_{u}, \mathbf{q}_{u} \mathbb{P}^{0} \mathbf{q}_{v}, \ldots, \mathbf{q}_{w} \mathbb{P}^{0} \mathbf{q}_{s}$, then $\mathbf{q}_{t}$ is strictly revealed preferred to $\mathbf{q}_{s}$ $\mathbf{q}_{t} \mathbf{P} \mathbf{q}_{s}$. The analogous definition again holds for the indirect strict revealed preference relation.

\section{Generalised Axiom of Revealed Preference (GARP). If $\mathbf{q}_{t} \mathbb{R} \mathbf{q}_{s}, \quad$ then $\mathbf{p}_{s}^{\prime} \mathbf{q}_{t} \geq x_{s}$}

GARP can be applied to set identify rational demands without any functional form assumptions on preferences beyond nonsatiation of the utility function. Intuitively, not all bundles that are affordable at a new budget are consistent (i.e. jointly satisfy GARP) with observed demand behaviour at budgets $t=\{1, \ldots, T\}$. The revealed preference approach relies on this consistency requirement to bound demand predictions. Each element of the set of predictions identified by revealed preference arguments can be jointly rationalised with past observations by a monotonic, concave, and non-degenerate utility function. ${ }^{4}$

This approach to demand prediction is well developed when interest lies in predicting behaviour at a single new budget of interest, i.e. $\mathscr{P}=\{T+1\}$. The set of rational demands that are recovered by revealed preference arguments is referred to as the 'support set', $S_{T}^{V}\left(\mathbf{p}_{T+1}, x_{T+1}\right)$, where the superscript $V$ denotes 'Varian' and the subscript $T$ is to reflect that predictions are made on the basis of observed demand behaviour at budgets $t=\{1, \ldots, T\}$. Any element of $S^{V}$ satisfies GARP in union with observed past consumption choices; any demand in the set's complement violates GARP.

Varian Support Set. Given the set of observations $\left\{\mathbf{p}_{t}, \mathbf{q}_{t}\right\}_{t=1, \ldots, T}$ and a new budget, $\left\{\mathbf{p}_{T+1}, x_{T+1}\right\}$, the

\footnotetext{
${ }^{4}$ Monotonicity of the utility function is a maintained assumption throughout this paper and ensures that recovered demands lie on budget hyperplanes.
} 
Varian support set is defined as:

$$
S_{T}^{V}\left(\mathbf{p}_{T+1}, x_{T+1}\right)=\left\{\begin{aligned}
& \mathbf{q}_{T+1} \geq \mathbf{0} \\
\mathbf{q}_{T+1}: & \mathbf{p}_{T+1}^{\prime} \mathbf{q}_{T+1}=x_{T+1} \\
& \left\{\mathbf{p}_{t}, \mathbf{q}_{t}\right\}_{t=1, \ldots, T+1} \quad \text { satisfies GARP }
\end{aligned}\right.
$$

The first and second constraints simply impose nonnegativity and budget exhaustion. The final condition imposes GARP. To give a simple example, imagine that we observe an individual's demand behaviour at the budgets $t=1,2$ :

$$
\begin{aligned}
& \mathbf{p}_{1}=\left[\begin{array}{l}
2 \\
1
\end{array}\right], \quad x_{1}=1, \quad \mathbf{q}_{1}=\left[\begin{array}{l}
0.3 \\
0.4
\end{array}\right] \\
& \mathbf{p}_{2}=\left[\begin{array}{l}
1 \\
2
\end{array}\right], \quad x_{2}=1, \quad \mathbf{q}_{2}=\left[\begin{array}{l}
0.4 \\
0.3
\end{array}\right]
\end{aligned}
$$

We want to predict behaviour at the new price regime:

$$
\mathbf{p}_{3}=\left[\begin{array}{l}
1.5 \\
1.2
\end{array}\right] \quad, \quad x_{3}=1
$$

Figure 1 gives the prediction problem graphically. GARP restricts counterfactual demands to lie on the line segment AB. Demanding strictly less than 0.2 units of $q^{1}$ at the new budget is ruled out because for any such demand, $\mathbf{q}_{3} \mathbb{R} \mathbf{q}_{1}$ but $\mathbf{p}_{1}^{\prime} \mathbf{q}_{3}<x_{1}$. Analogously, demanding strictly more than 0.44 units of $q^{1}$ is ruled out because for any such demand, $\mathbf{q}_{3} \mathbb{R} \mathbf{q}_{2}$ but $\mathbf{p}_{2}^{\prime} \mathbf{q}_{3}<x_{2}$.

The support set is convex, endowing it with a number of convenient properties for applied work. ${ }^{5}$ Further, the constraints defining membership of the support set are linear in unknowns and thus rational counterfactual demands can be easily recovered using linear programming methods (Varian 1982). This simplicity and practical convenience makes the approach convenient for applied work. With consumer panel data, observed individual demands can be used to bound counterfactual demands on an agent-by-agent basis (Adams 2014).

\footnotetext{
${ }^{5}$ See Proposition 2 of Blundell, Browning, and Crawford (2003) for a proof of this.
} 
Figure 1: Varian Support Set

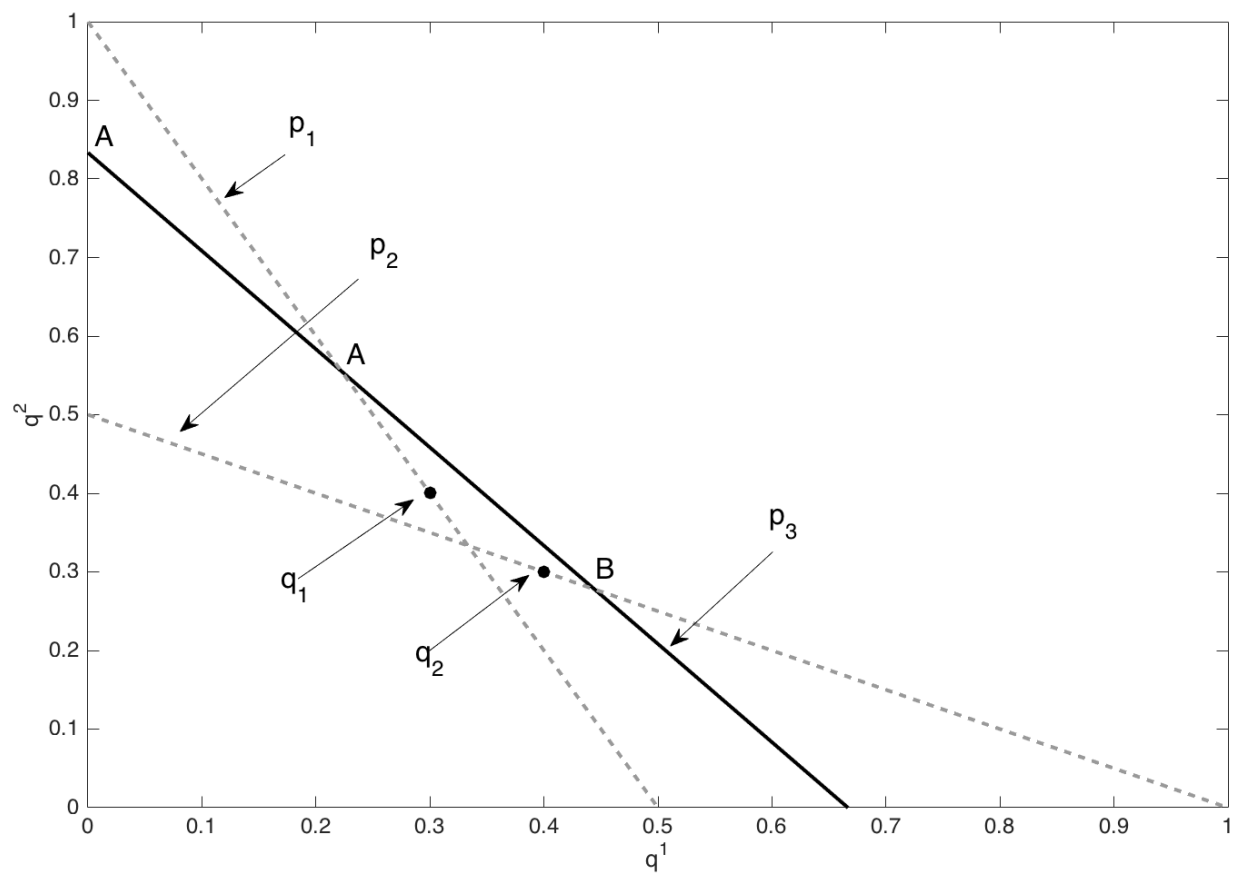

Figure notes: Demands $\mathbf{q}_{1}$ and $\mathbf{q}_{2}$ are observed at prices $\mathbf{p}_{1}$ and $\mathbf{p}_{2}$ respectively. Consistency with GARP constrains demand behaviour at the new budget $\mathbf{p}_{3}$ to the segment $\mathrm{AB}$. 
However, these techniques are also applied to aggregate and repeated cross section data, using conditional means or quantiles to bound predictions at new prices of interest (Varian 1982; Blundell, Browning, and Crawford 2008; Blundell, Kristensen, and Matzkin 2014).

\subsection{Recent Developments: E-Bounds}

Despite the approach's simplicity and its liberation from ad hoc functional form choices, the revealed preference approach to demand prediction faces problems. An oft levied criticism is that the bounds on demand responses yielded by the approach are too wide to be useful for any practical purpose. When few observed budgets are revealed worse to a new budget of interest, and when few hyperplanes intersect with the counterfactual budget of interest, revealed preference conditions lack bite and the bounds on demand predictions may be very wide.

In response to such criticisms, revealed preference techniques are increasingly used alongside estimated Engel curves to tighten the bounds on counterfactual demand predictions. Blundell, Browning, and Crawford (2008) were the first to show how one could use Engel curves to 'control' for budget variation in the data, and achieve the best bounds on nonparametric counterfactual demand predictions for the representative consumer. ${ }^{6}$ Rather than employ, for example, demands evaluated at mean income to bound expected demands at new budgets of interest, one instead estimate Engel curves in each of the $t=1, \ldots, T$ cross-sections and evaluates demands at a carefully chosen set of income levels, intersection incomes, for the purpose of bounding predictions at a new budget of interest.

Intersection Incomes. The intersection income $\tilde{x}_{t}$, for $t \in\{1, \ldots, T\}$ is the maximal income for which $\mathbf{q}_{T+1} \mathbb{R}^{0} \mathbf{q}_{t}\left(\tilde{x}_{t}\right)$, with $\mathbf{p}_{T+1}^{\prime} \mathbf{q}_{T+1}=x_{T+1}$.

Figure 2 depicts the key components of the approach. Rather than use the Marshallian demands at, for example, mean income, $\mathbf{q}_{1}\left(\bar{x}_{1}\right)$ and $\mathbf{q}_{2}\left(\bar{x}_{2}\right)$, to bound demands at $\left\{\mathbf{p}_{3}, x_{3}\right\}$, demands evaluated at the 'intersection incomes', $\mathbf{q}_{1}\left(\tilde{x}_{1}\right)$ and $\mathbf{q}_{2}\left(\tilde{x}_{2}\right)$, are employed, facilitating a refinement of the support set.

\footnotetext{
${ }^{6}$ See Blundell, Browning, Cherchye, Crawford, De Rock, and Vermeulen (2015) for the extension of the approach that also imposes transitivity.
} 
Figure 2: E-Bounds Support Set

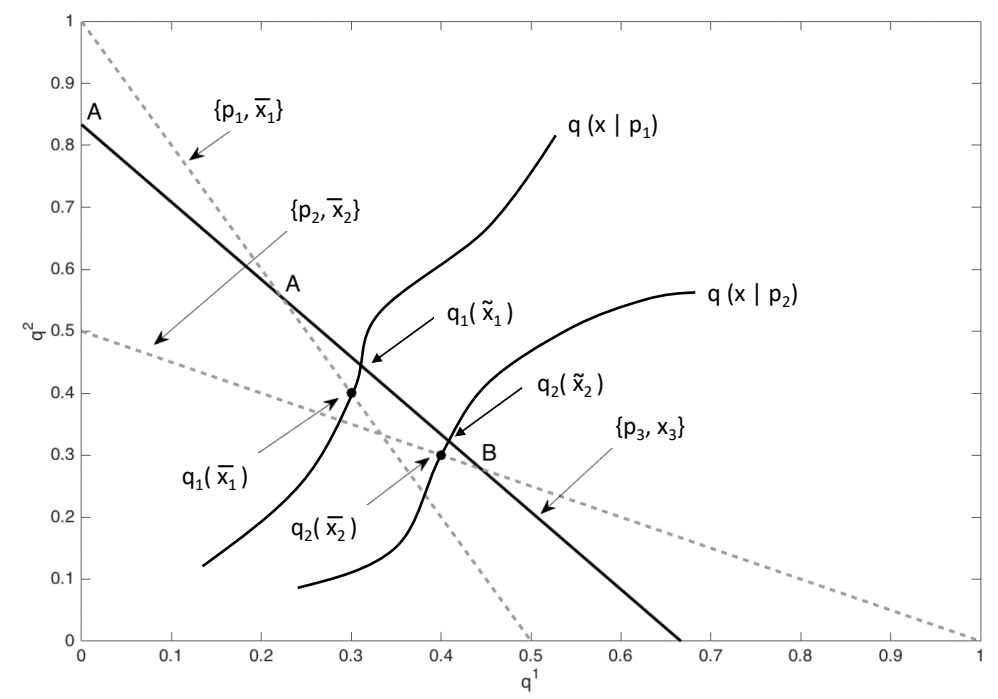

Figure notes: By using demands evaluated at intersection incomes, $\mathbf{q}_{1}\left(\widetilde{x}_{1}\right)$ and $\mathbf{q}_{2}\left(\widetilde{x}_{2}\right)$, one facilitates a refinement in the bounds on demand behaviour at $\left\{\mathbf{p}_{3}, x_{3}\right\}$.

The e-bound support set, $S_{T}^{e}\left(\mathbf{p}_{T+1}, \mathbf{q}_{T+1}\right)$, is then defined as:

E-Bound Support Set. Given the set of observed prices and intersection demands $\left\{\mathbf{p}_{t}, \mathbf{q}_{t}\left(\widetilde{x}_{t}\right)\right\}_{t=1, \ldots, T}$ and a new budget, $\left\{\mathbf{p}_{T+1}, x_{T+1}\right\}$, the support set is defined as:

$$
S^{e}\left(\mathbf{p}_{T+1}, x_{T+1}\right)=\left\{\begin{array}{cl} 
& \mathbf{q}_{T+1} \geq \mathbf{0} \\
\mathbf{q}_{T+1}: & \mathbf{p}_{T+1}^{\prime} \mathbf{q}_{T+1}=x_{T+1} \\
& \left\{\mathbf{p}_{1}, \ldots, \mathbf{p}_{T}, \mathbf{p}_{T+1}, \mathbf{q}_{1}\left(\widetilde{x}_{1}\right), \ldots, \mathbf{q}_{T}\left(\widetilde{x}_{T}\right), \mathbf{q}_{T+1}\right\} \text { satisfy GARP. }
\end{array}\right.
$$

Blundell, Browning, and Crawford (2008) show that e-bounds can yield very tight predictions on counterfactual demands, greatly enhancing the potential utility of revealed preference prediction exercises. The methodology has also been extended to impose transitivity (Blundell, Browning, Cherchye, Crawford, De Rock, and Vermeulen 2015) and to allow for richer forms of consumer heterogeneity. Blundell, Kristensen, and 
Matzkin (2014) allow for non-additive heterogeneity, adapting the technique to bound conditional quantiles predictions.

\section{The Need for Mutual Consistency}

The greater informativeness of e-bounds, and the fact that they can be applied to a wider variety of data sets, has rendered the revealed preference approach much more attractive. However, in applying the e-bound methodology to real-world data sets, an issue has arisen: estimated intersection demands, $\mathbf{q}_{t}\left(\widetilde{x}_{t}\right)$, do not always satisfy GARP themselves. This is a key problem because unless these demands satisfy rationality they cannot be used to bound rational counterfactual demands at new budgets of interest. As far as the author is aware, this issue has arisen in all applications of the methodology to date. Note that estimated intersection demands could be irrational simply due to sampling variation rather than any irrationality on the part of individual consumers or model misspecification. Indeed, in the limit, given that the demand function satisfies a boundary condition and is income-Lipschitzian, the revealed preference constraints do not bind (Mas-Colell 1978). However, there is no reason why this should be the case in finite samples.

The key difficulty that researchers have in imposing rationality on intersection demands is that there is no simple method for imposing mutual consistency or joint rationality of demand predictions. Imposing rationality on a set of demand predictions, such as is required for imposing GARP on the set of intersection demands used to construct e-bounds, is not addressed by Varian (1982). In the traditional consumer panel setting in which the revealed preference approach is well established, a consumer's past demand observations are used to bound a single counterfactual demand or consistency is imposed on a prediction-by-prediction basis.

The difficulty of imposing rationality on a set of estimated demands in the context of nonparametric demand estimation has also been recognised in the literature. For example, Blundell, Horowitz, and Parey (2012) and Blundell, Horowitz, and Parey (2017) estimate the demand function for gasoline subject to the Slutsky condition on a grid of budget points but do not impose that the Slutsky condition holds across points on the grid. While theory consistency constraints may be imposed for a number of reasons beyond ensuring rationality of predictions (for example, decreasing the variance of nonparametric estimates and improving out- 
of-sample extrapolation properties — see Matzkin (1994) for a review), rationality is an important property for demand predictions if they are to be used for welfare analysis. ${ }^{7}$ Further, even if taking a traditional revealed preference approach, using an observed consumer panel to directly bound counterfactuals, applied problems may require demand behaviour to be forecast at multiple budgets. For example, researchers may be interested in comparing behavioural responses across a number of different policy reforms simultaneously.

\subsection{The Sufficient Support Set}

Imposing consistency on a prediction-by-prediction basis is not sufficient for rationality of a set of predictions because it does not require that these predicted demands jointly satisfy GARP. To illustrate this in the context of the traditional Varian approach, let us return to the simple example from the last section. In

Figure 3, membership of $S_{2}^{V}\left(\mathbf{p}_{3}, x_{3}\right)$ constrains demand responses at $\left\{\mathbf{p}_{3}, x_{3}\right\}$ to the line segment $A B$ as before. We now, however, also predict demand at $\left\{\mathbf{p}_{4}, x_{4}\right\}$ :

$$
\mathbf{p}_{4}=\left[\begin{array}{l}
1.2 \\
1.5
\end{array}\right] \quad, \quad x_{4}=1
$$

Membership of $S_{2}^{V}\left(\mathbf{p}_{4}, x_{4}\right)$ constrains demand responses at $\left\{\mathbf{p}_{4}, x_{4}\right\}$ to the segment $C D$.

Yet, while the predictions in $S_{2}^{V}\left(\mathbf{p}_{3}, x_{3}\right)$ and $S_{2}^{V}\left(\mathbf{p}_{4}, x_{4}\right)$ are consistent with observed demands, they are not all consistent with one another. Formally, not all elements of the Cartesian product of these support sets satisfy revealed preference. In Figure 3, demands at OB are not mutually consistent with demands at OC. Imposing rationality on a budget-by-budget basis is necessary but not sufficient for consistency of all predictions with GARP; additional restrictions are required to ensure that predictions are themselves jointly rational.

In what follows, let the support set when demands are recovered at multiple budgets, and at which mutual consistency of predictions is imposed, be referred to as the 'sufficient support set', $S_{T}^{S}$.

Sufficient Support Set. Given the set of budgets $\mathscr{B}=\{1, \ldots, B\}$, for which demand behaviour is observed

\footnotetext{
${ }^{7}$ If, for example, nonparametric demand predictions jointly fail rationality, methods applied to these predictions to estimate consumer surplus and there welfare metrics will suffer from path dependency.
} 
Figure 3: Multiple Predictions

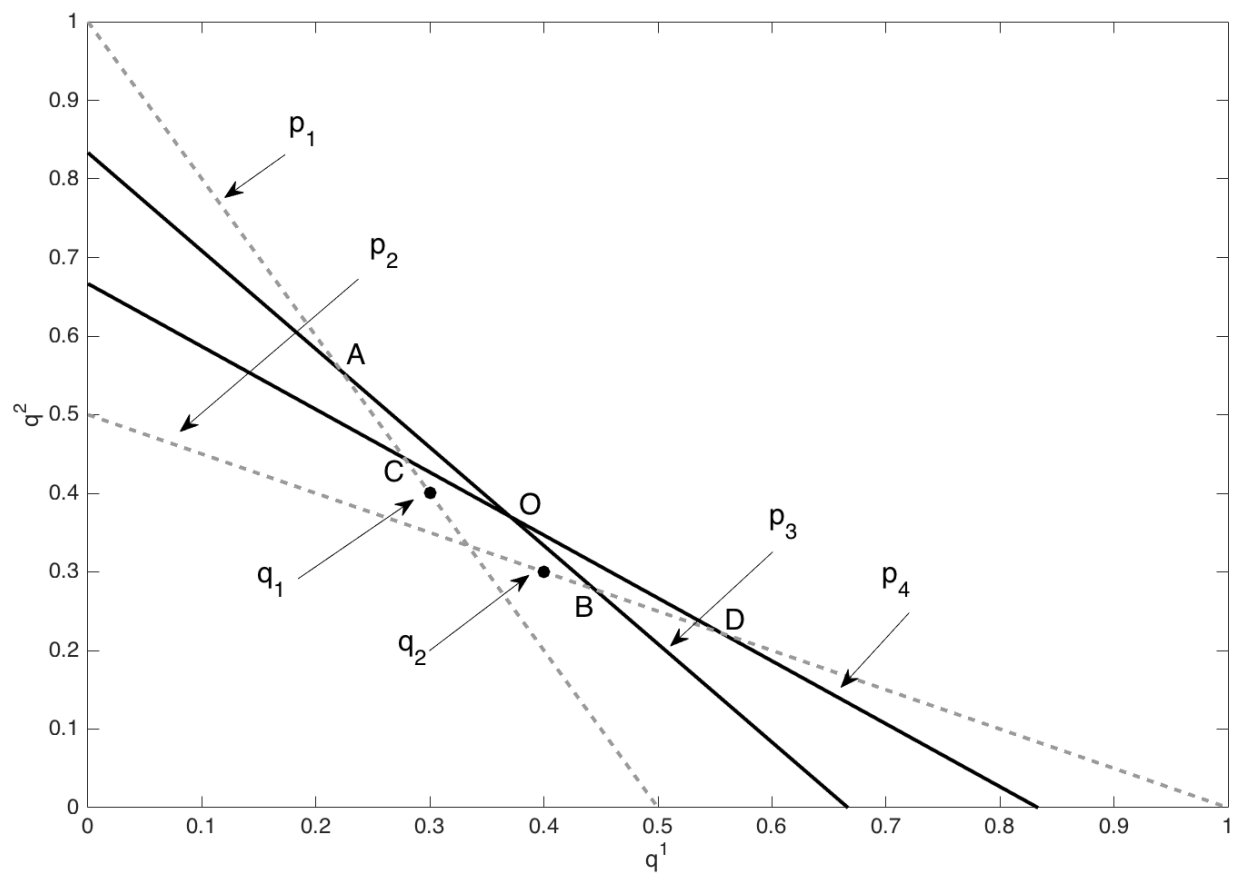

Figure note: Demands $\mathbf{q}_{1}$ and $\mathbf{q}_{2}$ are observed at budgets $\left\{\mathbf{p}_{1}, x_{1}\right\}$ and $\left\{\mathbf{p}_{2}, x_{2}\right\}$. We want to bound predictions at $\left\{\mathbf{p}_{3}, x_{3}\right\}$ and $\left\{\mathbf{p}_{4}, x_{4}\right\}$. Demands in patch $O B$ and patch $O C$ fail GARP. 
for the subset $\mathscr{D}=\{1, \ldots, T\}$ and is to be predicted over the subset $\mathscr{P}=\{T+1, \ldots, B\}$, the sufficient support set is defined as:

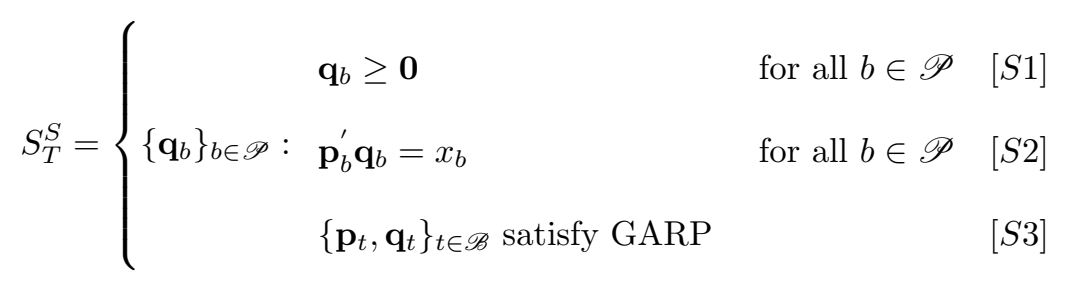

\subsection{Intersection Demands \& Rational Nonparametric Demand Systems}

In the context of e-bounds (i.e. revealed preference predictions using Engel curves), the issue of multiple prediction arises in the estimation of the $T$ intersection demands in addition to the problem of predicting demands at multiple new price vectors of interest. Imposing rationality on a single Engel curve given a cross section without price variation is trivially satisfied without further functional form restrictions. ${ }^{8}$ However, estimating Engel curves at a number of different price vectors to recover a set of intersection demands leads to a similar problem as when bounding counterfactual demands at multiple budgets of interest. Figure 4 gives one such example of this. Given (estimated) Engel curves, $\mathbf{q}\left(x \mid \mathbf{p}_{1}\right)$ and $\mathbf{q}\left(x \mid \mathbf{p}_{2}\right)$ as depicted, predicted intersection demands at the new budget of interest $\left\{\mathbf{p}_{3}, x_{3}\right\}$ fail GARP. If taking any one Engel curve or intersection demand individually, this issue of joint rationality would not arise.

Estimating nonparametric demand systems subject to a rationality constraint is a closely related problem. While several methods exist for imposing Slutsky symmetry upon nonparametric demand systems (Haag, Hoderlein, and Pendakur 2009; Blundell, Horowitz, and Parey 2012; Blundell, Horowitz, and Parey 2017), this is only necessary but not sufficient for rationality. For rationality, the Slutsky matrix must also be negative semidefinite.

\subsection{Nonconvexity}

Different strategies have been used to cope with the irrationality of estimated intersection demands when constructing e-bounds. One simple strategy involves searching for contiguous periods of revealed-preferenceconsistent intersection demands and discarding those responsible for violations (Blundell, Browning, and

\footnotetext{
${ }^{8}$ Any pattern of behaviour could be considered rational as budget hyperplanes will not cross and revealed preference restrictions will not bind.
} 
Figure 4: Irrational Intersection Bounds

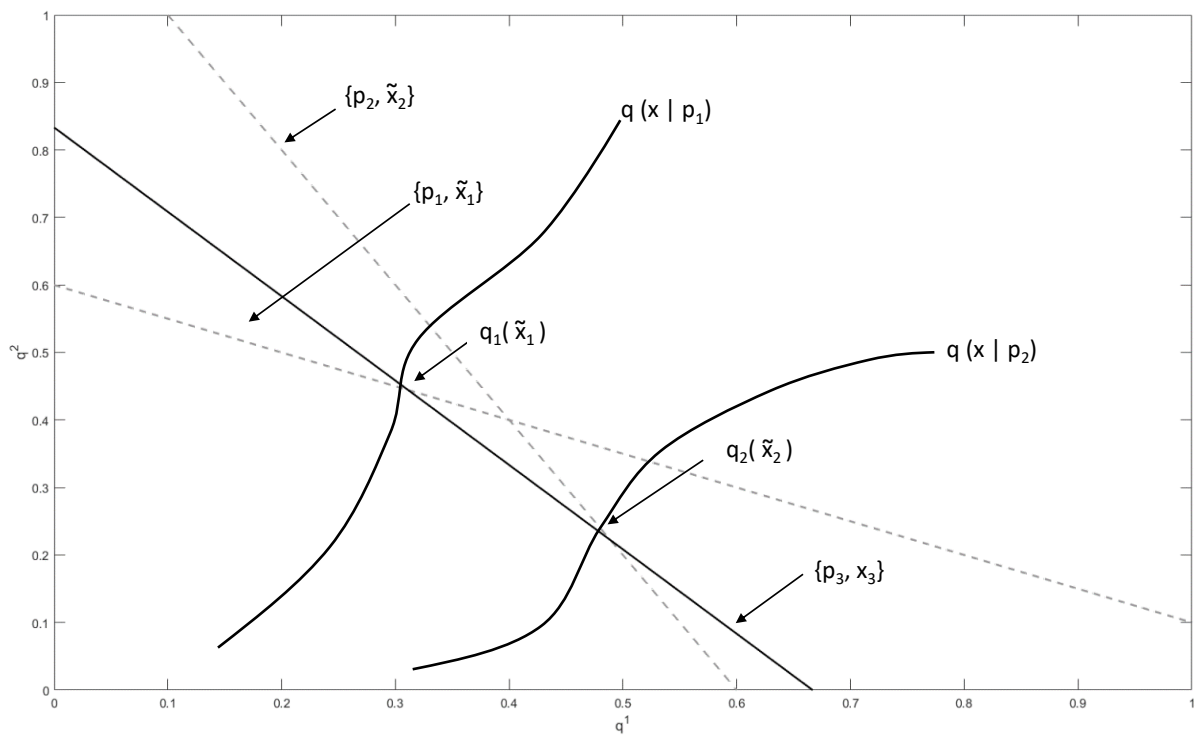

Figure notes: Intersection demands, $\mathbf{q}_{1}\left(\widetilde{x_{1}}\right)$ and $\mathbf{q}_{2}\left(\widetilde{x_{2}}\right)$, violate GARP. Therefore, no demand behaviour at $\mathbf{p}_{3}, x_{3}$ will satisfy revealed preference constraints in union with these intersection demands.

Crawford 2008). The drawback of such an approach is that bounds may remain wide if many intersection demands are dropped. A popular alternative has been to perturb unconstrained intersection demands, $\widehat{\mathbf{q}}_{t}\left(\mathbf{p}_{t}, \widetilde{x}_{t}\right)$ such that they jointly satisfy GARP (Blundell, Browning, and Crawford 2008). Constrained intersection demands are then defined as the solution to a constrained non-linear least squares problem

$$
\left\{\hat{\mathbf{q}}_{t}^{C}\right\}_{t=1, \ldots, T}=\arg \min _{\left\{\mathbf{q}_{t}\right\}_{t=1, \ldots, T}} \sum_{t=1}^{T}\left(\mathbf{q}_{t}-\widehat{\mathbf{q}}_{t}\left(\widetilde{x}_{t}\right)\right)^{2}
$$

subject to $\left\{\hat{\mathbf{q}}_{t}^{C}\right\}_{t=1, \ldots, T}$ jointly satisfying GARP, i.e. mutual consistency of predictions. ${ }^{9}$

This is a computationally challenging optimisation problem given the nonconvexity of the solution set, which can create significant estimation difficulties:

“... in fact, the great watershed in optimisation isn't between linearity and nonlinearity, but between convexity and nonconvexity"

\footnotetext{
${ }^{9}$ This was done by imposing the constraint that the Afriat efficiency index of the set of intersection demands is zero using non-linear optimisation techniques (Blundell, Browning, and Crawford 2008). See Blundell, Kristensen, and Matzkin (2014) for an alternative two-step estimation strategy to estimate revealed preference consistent income expansion paths.
} 
To illustrate this issue, let us return again to our illustrative Varian-type example. As there are only two goods, every feasible budget share specification at the new budgets, $\left\{\mathbf{p}_{3}, x_{3}\right\}$ and $\left\{\mathbf{p}_{4}, x_{4}\right\}$, can be represented in a two-dimensional diagram as in Figure 5. Demand predictions that are consistent with revealed preference, and thus are elements of the sufficient support set, are given in light grey. Budget shares that are elements of the Cartesian product of the Varian support sets but jointly violate revealed preference are given in dark grey. This makes it clear that the sufficient support set is nonconvex. This precludes the construction of the support set by linear programming methods, increasing the complexity of the characterisation of the support set and complicating optimisation over its elements. ${ }^{11}$

Figure 5: Varian Support Set

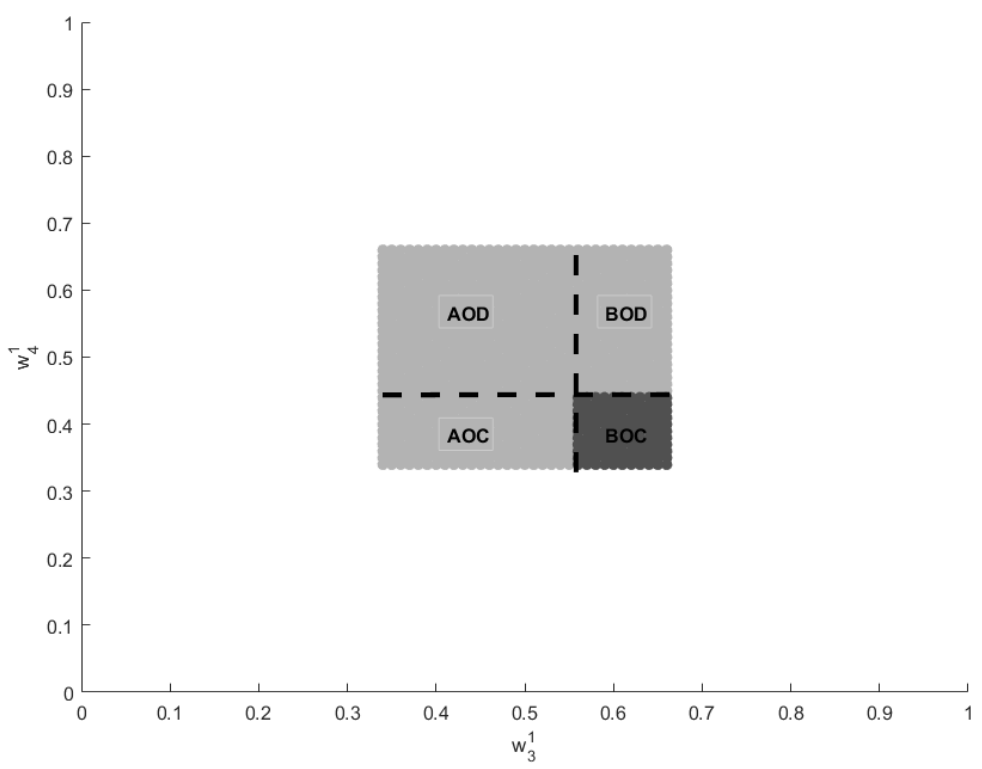

The light grey region of panel corresponds to budget shares in the sufficient support set of the prediction problem given in Figure 3 .

If the Varian support sets of any new budgets intersect at an interior point, not all combinations of demands drawn from each Varian support set will mutually satisfy rationality and the sufficient support set is nonconvex:

\footnotetext{
${ }^{10}$ R. Tyrrell Rockafellar (1993) "Lagrange Multipliers and Optimality", SIAM Review 35 (2), 183-238.

${ }^{11}$ Any linear program is a convex optimisation problem (see, for example, Boyd and Vandenberghe (2004)).
} 
Proposition $1 S_{T}^{S}$ is not convex if there exist budgets $a, b \in \mathscr{P}$ at which $\exists \tilde{\mathbf{q}} \in \mathbb{R}_{++}^{K}$ such that $\tilde{\mathbf{q}} \in S_{T}^{V}\left(\mathbf{p}_{a}, x_{a}\right)$ and $\tilde{\mathbf{q}} \in S_{T}^{V}\left(\mathbf{p}_{b}, x_{b}\right)$.

Thus, the issue of non-convexity does not arise when one is only changing a single price while keeping the budget constant; in this case, budget hyperplanes will not intersect at an interior point.

Note that the requirement of mutual consistency serves to restrict combinations of demand predictions at new budgets of interest. Indeed, there will exist paths of rational demands over each new budget of interest such that each element of every Varian support set features in $S_{T}^{S}$ (just not every combination of every element will feature).

Proposition 2 For each $\mathbf{q}_{a} \in S_{T}^{V}\left(\mathbf{p}_{a}, x_{a}\right)$, there will exist a set of demand predictions $\left\{\mathbf{q}_{b}\right\}_{b \in \mathscr{P} / a}$ such that $\left\{\mathbf{q}_{a},\left\{\mathbf{q}_{b}\right\}_{b \in \mathscr{P} / a}\right\} \in S_{T}^{S}$

Proposition 2 implies that it is not possible for every combination of demand from the Varian support sets to be jointly irrational. Only a proper subset of the Cartesian product of Varian support sets fails the requirement of mutual consistency. ${ }^{12}$

The aim of the rest of this paper is to develop a practical characterisation of the sufficient support set for empirical work that can be applied to bound demand predictions across a set of budgets of interest, to estimate rational intersection demands, and to impose a sufficient condition for rationality of predictions on nonparametric regression estimates. Whilst unable to remove the fundamental nonconvexity of the problem, I will provide an elegant formulation of the problem for which there is a large and powerful machinery for solving.

\footnotetext{
${ }^{12}$ Where $\varnothing$ is considered a proper subset. Considering a sequential characterisation of the support set helps to demonstrate this point. (I would like to thank a referee for noticing this point.) Imagine selecting a demand at the first budget of interest, $T+1$, from the associated Varian support set, $S_{T}^{V}$. Then, select a prediction from the $T+2$ budget from the Varian support set conditional on the prediction at $T+1, S_{T+1}^{V}$.
}

$$
S^{S}= \begin{cases} & \mathbf{q}_{T+1} \in S_{T}^{V}\left(\mathbf{p}_{T+1}, x_{T+1}\right) \\ \left\{\mathbf{q}_{b}\right\}_{b \in \mathscr{P}}: & \vdots \\ & \mathbf{q}_{T+2} \in S_{T+1}^{V}\left(\mathbf{p}_{T+2}, x_{T+2}\right) \\ & \mathbf{q}_{B} \in S_{B-1}^{V}\left(\mathbf{p}_{B}, x_{B}\right)\end{cases}
$$




\section{A Mixed Integer Characterisation}

The sufficient support set cannot in general be characterised by a linear programme given the interdependence of rational demands across new budgets. However, a mixer integer linear programming (MILP) representation of the necessary and sufficient constraints that define $S_{T}^{S}$ can be formulated. ${ }^{13}$ The field of mixed integer programming has undergone "remarkable" improvements in recent years (Lima and Grossmann 2011). While the MILP formulation does not remove the fundamental nonconvexity of our problem, a powerful set of algorithms are available in standard software for solving problems of this type.

Proposition 3: MILP Representation of $S_{T}^{S}$ Given the set of budgets $\mathscr{B}$, for which demand behaviour is observed for the subset $\mathscr{D} \subset \mathscr{B}$ and is to be predicted over the subset $\mathscr{P}=\mathscr{B} / \mathscr{D}$, the MILP representation of the sufficient support set is defined as:

$$
S^{S}=\left\{\begin{aligned}
\mathbf{q}_{b} & \geq 0 & \text { for all } b \in \mathscr{P} & {[1] } \\
\mathbf{p}_{b}^{\prime} \mathbf{q}_{b} & =x_{b} & \text { for all } b \in \mathscr{P} & {[2] } \\
\mathbf{p}_{b}^{\prime} \mathbf{q}_{a} & >\left(1-R_{b a}\right) x_{b} & \text { for all } a \neq b \in \mathscr{B} & {[3] } \\
\left.R_{a b}+\mathbf{q}_{b}\right\}_{b \in \mathscr{P}} & \leq 1+R_{a c} & & \text { for all } a \neq b \neq c \in \mathscr{B} \\
\mathbf{p}_{b}^{\prime} \mathbf{q}_{a} & \geq R_{a b} x_{b} & \text { for all } a \neq b \in \mathscr{B} & {[5] } \\
R_{a b} & =\{0,1\} & \text { for all } a \neq b \in \mathscr{B} & {[6] }
\end{aligned}\right.
$$

Constraints [1] through [5] are linear in unknowns and provide an operational methodology with which practically to characterise the sufficient support set. Constraint [6] links the integer variable $R_{a b}$ to the revealed preference relation. $\mathbf{q}_{a} \mathbb{R} \mathbf{q}_{b}$ is computationally represented by $R_{a b}=1$.

Constraints [1] and [2] impose that predictions respect the standard nonnegativity and adding up requirements. Constraint [3] defines the direct revealed preference relation, imposing the requirement that $\mathbf{q}_{b} \mathbb{R} \mathbf{q}_{a}$, i.e. $R_{b a}=1$, if $\mathbf{p}_{b}^{\prime} \mathbf{q}_{a} \leq x_{b}$, thereby defining the direct revealed preference relation. To see this note that if

\footnotetext{
${ }^{13}$ Cherchye, De Rock, Sabbe, and Vermeulen (2008) characterise a necessary condition for collective rationality using a MILP representation.
} 
$R_{b a}=0$, Constraint [3] evaluates as $\mathbf{p}_{b}^{\prime} \mathbf{q}_{a}>x_{b}$, which is a contradiction when $\mathbf{p}_{b}^{\prime} \mathbf{q}_{a} \leq x_{b}$. Constraint [4] imposes transitivity on the revealed preferred relation: for any budget $a$ that is preferred to budget $b$, that is revealed preferred to budget $c, R_{b c}=1$, it must be the case that $a$ is preferred to $c, R_{a c}=1$.

Constraint [5] imposes GARP: for any bundle $\mathbf{q}_{a}$ that is preferred to a bundle $\mathbf{q}_{b}$ (and thus $R_{a b}=1$ ), it must be the case that $\mathbf{q}_{a}$ is more expensive than $x_{b}$.

To illustrate how demands in the region $B O C$ in Figure 5 fail these conditions, imagine that at $\left\{\mathbf{p}_{3}, x_{3}\right\}$ we draw some $\mathbf{q}_{3} \in O B$ and at $\left\{\mathbf{p}_{4}, x_{4}\right\}$ we draw some $\mathbf{q}_{4} \in C O$. We have that $\mathbf{p}_{3}^{\prime} \mathbf{q}_{4}<x_{3}$ and $\mathbf{p}_{4}^{\prime} \mathbf{q}_{3}<x_{4}$. Thus, for Constraint [3] to be satisfied $R_{34}=1$ and $R_{43}=1$. However, this leads to a contradiction at Constraint [5], which requires $R_{34}=0$ and $R_{43}=0$. For demands in the sufficient support set, a specification for the binary variables can be found such that the MILP constraints are satisfied.

Cost Efficiency Indices In some empirical applications of revealed preference methods, small deviations from GARP are permitted. The most common way of allowing for small deviations of demand from GARP is to define the Afriat Critical Cost Efficiency Index, $0<e \leq 1$ (see Andreoni, Gillen, and Harbaugh (2011) for a review). The lower is $e$, the greater the permitted inefficiency of consumer choice and the larger the tolerable violations of GARP. The MILP constraints are easily modified to allow for some inefficiency in demand predictions such that GARP does not hold precisely. If one wanted to allow for a level of inefficiency $e$, one would replace Constraint [5], the GARP constraint, with:

$$
\mathbf{p}_{b}^{\prime} \mathbf{q}_{a} \geq e R_{a b} x_{b}
$$

where $0<e \leq 1$ gives the pre-specified inefficiency level.

\subsection{Efficiency Enhancements}

Transitivity The MILP representation above is intuitive given that it links directly to the definition of GARP and the transitivity of the direct revealed preference relation. However, it involves $O\left(B^{3}\right)$ constraints, which quickly results in large formulations that can be hard to solve. Following Nobibon, Cherchye, Crama, Demuynck, De Rock, and Spieksma (2016), harnessing insights from graph theory facilitates a more compact 
representation involving only $O\left(B^{2}\right)$ constraints. Specifically, Proposition 3 of Nobibon, Cherchye, Crama, Demuynck, De Rock, and Spieksma (2016) can be applied to replace Constraint [4] with Constraints [4a] and $[4 \mathrm{~b}] .^{14}$

$$
\begin{aligned}
& u_{a}-u_{b}<R_{a b} \quad \text { for all } a \neq b \in \mathscr{B} \quad[4 a] \\
& R_{a b}-1 \leq u_{a}-u_{b} \quad \text { for all } a \neq b \in \mathscr{B} \quad[4 b]
\end{aligned}
$$

Under this formulation, the $R_{a b}$ variables come to represent the ordering of utility levels, i.e. $R_{a b}=1$ iff $u_{a} \geq u_{b}$, rather than the revealed preference relation.

While this new formulation involves $2 B$ additional continuous variables, there are only $O\left(B^{2}\right)$ constraints. For even moderately large data sets, this significantly reduces the size of the problem bringing significant computation time savings. In the context of testing the collective model of household consumption, Nobibon, Cherchye, Crama, Demuynck, De Rock, and Spieksma (2016) find that switching to the more efficient transitivity representation facilitates a tripling in the size of computationally feasible models and a large reduction in computation time: testing for rationalisability on a data set of 60 observations took approximately 12 minutes with the collective model equivalent of Constraint [4] but only a matter of seconds with the equivalents of $[4 \mathrm{a}]$ and $[4 \mathrm{~b}]$. Further, note that the computational burden of imposing transitivity is completely eliminated in 2-good problems. As first proven by Rose (1958), transitivity has no empirical content when $\mathrm{K}=2$. In this special case then, Constraint [4] may be dropped from the programming problem. ${ }^{15}$

Irrelevant Budget Comparisons As often recognised in applications of revealed preference methods, some budget comparisons are irrelevant for testing or imposing consistency with a particular model (Kitamura and Stoye 2017; Barseghyan, Coughlin, Molinari, and Teitelbaum 2018). In our context, mutual consistency of predictions does not need to be imposed across sets of non-intersecting new budgets. ${ }^{16}$ Let the undirected intersection relation between budgets $a, b$ be given as $I_{a b}=1$ if there is an intersection path between the

\footnotetext{
${ }^{14}$ Note that if one was interested in imposing the Strong Axiom of Revealed Preference, which rules out indifferences, only Constraint $4 \mathrm{a}$ is required. I would like to thank Thomas Demuynck for helpful conversations on this point.

${ }^{15}$ See Blundell, Browning, Cherchye, Crawford, De Rock, and Vermeulen (2015) for a further discussion of the complications caused by transitivity for testing and imposing SARP at a single budget.

${ }^{16}$ This is the same insight as in Kitamura and Stoye (2017), proof of Theorem 3.2. We discuss the connections between our approaches in more depth later in the section.
} 
budgets, and $I_{a b}=0$ otherwise. This can be constructed as follows. First, compute the intersection relation $I^{0}$, where:

$$
I_{a b}^{0}=I_{b a}^{0}=1 \quad \text { if } \quad \exists \mathbf{q}>\mathbf{0} \text { such that } \mathbf{p}_{a}^{\prime} \mathbf{q}=\mathbf{p}_{b}^{\prime} \mathbf{q},
$$

and $I_{a b}^{0}=I_{b a}^{0}=0$ otherwise, for $a \neq b$ and $a, b=\{1, \ldots, B\} .{ }^{17}$ Warshall's Algorithm (Warshall, 1962) can then be applied to compute the transitive closure of this relation to give $I_{a b}$.

Subsets of non-intersecting budgets $\mathscr{P}_{m}$ are defined such that within a subset there is a path between intersecting budgets $\left(\forall a, b \in \mathscr{P}_{m}, I_{a b}=1\right)$ but between subsets there is no path of intersecting budgets $\left(\forall a \in \mathscr{P}_{m}, b \in \mathscr{P}_{n}\right.$ with $\left.m \neq n, I_{a b}=0\right)$. Mutual consistency of predictions can then be independently imposed on each subset of new budgets $\mathscr{P}_{m}$ for joint rationality of the full set of predictions. That is, mutual consistency restrictions will never be binding for demands predicted at budgets in different subsets of non-intersecting budgets. ${ }^{18}$

Proposition 4 Define subsets $\mathscr{P}_{m}$, such that $\mathscr{P}=\cup_{m=1}^{M} \mathscr{P}_{m}$ and $\forall a, b \in \mathscr{P}_{m}, I_{a b}=1$ and $\forall c \in \mathscr{P}_{m}$, $d \in \mathscr{P}_{n}$ with $m \neq n, I_{c d}=0$. Then, the sufficient support set is defined as:

$$
S_{T}^{S}=\left\{\begin{array}{rlll}
\mathbf{q}_{b} & \in S_{T}^{V}\left(\mathbf{p}_{b}, x_{b}\right) & \text { for all } b \in \mathscr{P} & \\
\text { for } m=1, \ldots, M & & \\
\left\{\mathbf{q}_{b}\right\}_{b \in \mathscr{P}}: & >\left(1-R_{b a}\right) x_{b} & \forall a \neq b \in \mathscr{P}_{m} & {[2]} \\
\mathbf{p}_{b}^{\prime} \mathbf{q}_{a} \mathbf{q}_{a} & \geq R_{a b} x_{b} & \forall a \neq b \in \mathscr{P}_{m} & {[3]} \\
u_{a}-u_{b} & <R_{a b} & \forall a \neq b \in \mathscr{P}_{m} \cup \mathscr{D} & {[4 a]} \\
R_{a b}-1 & \leq u_{a}-u_{b} & \forall a \neq b \in \mathscr{P}_{m} \cup \mathscr{D} & {[4 b]}
\end{array}\right.
$$

where $R_{a b}=\{0,1\}$.

\footnotetext{
${ }^{17}$ This is easily constructed with standard linear programming methods.

${ }^{18}$ Proposition 4 is particularly useful if one is interested in predicting demands along a price path where only the relative price of good $k$ is varied. In this case, no budget in this set intersects with another at strictly positive quantities and so the prediction problem is easily solved using a series of independent linear programmes.
} 


\subsection{Connection to Alternative Approaches}

An alternative characterisation of the sufficient support set comes from viewing the multiple prediction problem as a discrete choice problem. Hoderlein and Stoye (2015) and Kitamura and Stoye (2017) ('KS') use the insight that with a finite set of budgets nonparametric economic theory does nothing more than constrain demand behaviour to belong to a discrete set of rational 'choice types' to construct tests for the Weak Axiom of Revealed Stochastic Preference and the Axiom of Revealed Stochastic Preference respectively. This section serves to build a connection to this work.

To connect this work to our setting, consider again the multiple prediction example given previously, and repeated at Figure 6 for the reader's convenience. In this example, KS's approach would define three rational choice types:

1. At $\left\{\mathbf{p}_{3}, x_{3}\right\}$ demand in patch $A O$ and at $\left\{\mathbf{p}_{4}, x_{4}\right\}$ demand in patch $C O$;

2. At $\left\{\mathbf{p}_{3}, x_{3}\right\}$ demand in patch $A O$ and at $\left\{\mathbf{p}_{4}, x_{4}\right\}$ demand in patch $O D$

3. At $\left\{\mathbf{p}_{3}, x_{3}\right\}$ demand in patch $O B$ and at $\left\{\mathbf{p}_{4}, x_{4}\right\}$ demand in patch $O D$.

However, demand at $O B$ and $O D$ is not a rational choice type. Note that rationality does not restrict where demand lies within the set of predictions consistent with a given choice type.

It is clear that the sufficient support set can be recast in terms of rational choice types: each element of the sufficient support set must be consistent with a rational choice type. This connection is explored formally in the Appendix. Why then is the MILP representation of the mutual consistency constraints novel and useful? Why not simply constrain predictions to be consistent with at least one rational choice type?

For certain purposes, application of the methodology developed by KS will be preferable: if there are only a small number of rational choice types (because budget hyperplanes rarely cross) or one wants to enumerate the set of all mutually consistent demand predictions, directly imposing consistency with a rational choice type using mixed integer programming methods may yield a smaller optimisation problem and/or more directly interpretable output. Further, if one's object is instead to test consistency with the Axiom of Revealed Stochastic Preference or to bound expected demands with a heterogeneous population of consumers then one should of course apply the methodology developed in Kitamura and Stoye (2017) and Hoderlein and Stoye 
Figure 6: Multiple Predictions

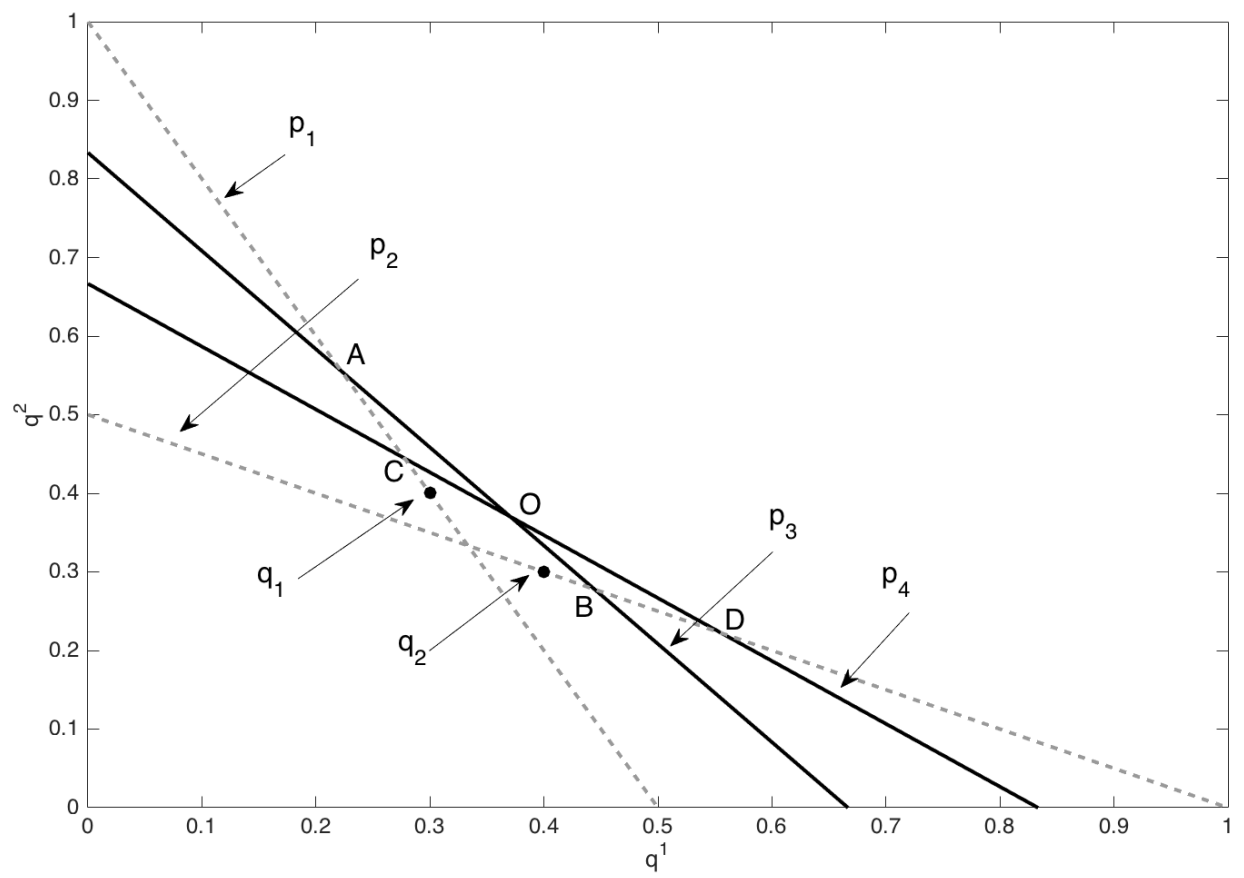

Figure note: Demands $\mathbf{q}_{1}$ and $\mathbf{q}_{2}$ are observed at budgets $\left\{\mathbf{p}_{1}, x_{1}\right\}$ and $\left\{\mathbf{p}_{2}, x_{2}\right\}$. We want to bound predictions at $\left\{\mathbf{p}_{3}, x_{3}\right\}$ and $\left\{\mathbf{p}_{4}, x_{4}\right\}$. Demands in patch $O B$ and patch $O C$ fail GARP. 
(2015).

However, for problems in which the main aim is to constrain demand predictions using moderately sized data sets, the formulation put forward in this paper will often yield a much less computationally burdensome procedure. To illustrate, if one was interested in constraining demand predictions at the seven budgets used in one of KS's empirical applications, the number of variables associated with a mixed integer programme to impose consistency with a given rational choice type would reach as high as 336,467 with 5 goods. ${ }^{19}$ Our preferred MILP formulation would, however, involve only 84 variables. ${ }^{20}$ Third, in addition to time savings for the optimisation itself, it is not necessary to encode the rational choice types prior to the optimisation step under the formulation in this paper. The computational benefits of the approach put forward in this paper over the method developed in Kitamura and Stoye (2017) for moderately sized prediction problems will be clear in the empirical application when computation times are compared directly.

\section{Empirical Application}

To illustrate the application of the rationality constraints developed in this paper, I impose mutual consistency of demand predictions in a nonparametric regression setting. The aim is to show applied researchers how the rationality constraints developed in this paper can be applied in practise and further to demonstrate the utility of the approach over alternative methods. Specifically I will apply the constraints to impose membership of the sufficient support set on intersection demand estimates, which are a necessary input to the e-bound methodology. Thus, I predict a set of jointly rational demand predictions at the intersection incomes using cross section data on consumer demand behaviour at different prices and income levels.

To proceed, I first outline my empirical specification and a mutual-consistency constrained kernel estimator of demand. I then describe the data and the standard freely available software that is used. Finally, I outline the results and provide evidence on how the estimator performs as I increase the number of budgets at which demand is predicted at.

\footnotetext{
${ }^{19}$ The set of budgets between 1989 and 1995 define 336,467 rational choice types.

${ }^{20}$ There are 70 constraints associated with KS's approach and 210 associated with the one put forward in this paper.
} 


\subsection{Rationality Constrained Kernel Estimator}

Intersection demands are gained by estimating Engel curves at a carefully chosen set of incomes, $\widetilde{x}_{t}$ for $t=1, \ldots, T$. We want to restrict this set of demand predictions to satisfy GARP, that is for the set of demand predictions to be an element of the sufficient support set. The data are observations $\left\{\mathbf{w}_{i}, x_{i}\right\}$ for $N$ randomly sampled individuals at each of the $t=1, . ., T$ price regimes $\mathbf{p}_{t}$, where $x_{i}$ and $\mathbf{w}_{i} \in \mathbb{R}_{+}^{K}$ give the income and budget shares of individual $i$ respectively. I take a simple specification for the Engel curve within a price regime $t$ :

$$
w_{i}^{k}=g_{t}^{k}\left(x_{i}\right)+\epsilon_{i}^{k}
$$

where, for each individual $i$, the error term satisfies $E\left(\varepsilon_{i}^{k} \mid x\right)=0$ and $\operatorname{var}\left(\varepsilon_{i}^{k} \mid x\right)=\sigma^{2}(x)$ for $k=1, \ldots, K-1$. The budget share of the $K^{t h}$ good is obtained by adding up: $g_{t}^{K}(x)=1-\sum_{k=1}^{K-1} g_{t}^{k}(x)$.

Fully nonparametric estimates of $g_{t}^{k}(\cdot)$ for $t=1, \ldots, T$ and $k=1, \ldots, K-1$, which are not constrained to satisfy rationality, can be obtained using the Nadaraya-Watson kernel estimator (Nadaraya 1964; Watson 1964): ${ }^{21}$

$$
\widehat{g}_{t}^{k}(x)=\frac{1}{N} \sum_{i=1}^{N} \frac{K_{h}\left(x-x_{i}\right) w_{i}^{k}}{\sum_{j=1}^{N} K_{h}\left(x-x_{j}\right)}
$$

where $K$, the kernel function, is a bounded, differentiable probability density function that is symmetric about zero and $h$ is the bandwidth parameter. Demand predictions at the intersection income levels, $\widetilde{x}_{t}$, the intersection demands, are then:

$$
\widehat{q}_{t}^{k}=\frac{\widetilde{x}_{t} \widehat{g}_{t}^{k}\left(\widetilde{x}_{t}\right)}{p_{t}^{k}}
$$

However, these estimated intersection demands are not necessarily mutually consistent and may fail GARP. To impose the rationality constraints defined by Proposition 4 on estimated demand predictions, I

\footnotetext{
${ }^{21}$ See Härdle (1990) for a discussion of the properties of the unconstrained estimator. Provided that the same bandwidth and kernel are used to estimate each $g^{k}(x)$, adding up will be automatically satisfied and there is no efficiency gain from combining equations; see Blundell, Browning, and Crawford (2003).
} 
follow Hall and Huang (2001) and Blundell, Horowitz, and Parey (2012). Specifically, I replace $\widehat{g}(\cdot)$ with the weighted estimator:

$$
\widehat{g}_{t}^{k, C}(x)=\frac{1}{N} \sum_{i=1}^{N} \delta_{i t} \frac{K_{h}\left(x-x_{i}\right) w_{i}^{k}}{\sum_{j=1}^{N} K_{h}\left(x-x_{j}\right)}
$$

where $\delta_{i t}$ are weakly positive weights that are chosen to ensure that estimated demands are mutually consistent and satisfy GARP. Let $\delta=\left[\delta_{1 t}, \ldots, \delta_{N t}\right]$. Formally, these weights are obtained by solving the optimisation problem:

$$
\min _{\delta_{1}, \ldots, \delta_{N}} \sum_{t=1}^{T} \sum_{i=1}^{N}\left(\delta_{i t}-1\right)^{2}
$$

subject to:

$$
\begin{array}{rlrl}
\frac{1}{N} \sum_{i=1}^{N} \delta_{i t} & =1 & \text { for } t=1, \ldots, T \\
\widehat{q}_{t}^{C, k} & =\frac{\widehat{g}_{t}^{k, C}\left(\widetilde{x}_{t}\right) \widetilde{x}_{t}}{p_{t}^{k}} & & \text { for } t=1, \ldots, T \\
\mathbf{p}_{t}^{\prime} \widehat{\mathbf{q}}_{t}^{C} & =\widetilde{x}_{t} & \text { for } t=1, \ldots, T \\
\mathbf{p}_{t}^{\prime} \widehat{\mathbf{q}}_{s}^{C} & >\left(1-R_{t s}\right) x_{t} & \text { for all } s \neq t \\
R_{s t}+R_{t u} & \leq 1+R_{s u} & \text { for all } s \neq t \neq u \\
\mathbf{p}_{t}^{\prime} \widehat{\mathbf{q}}_{s}^{C} & \geq R_{s t} x_{t} & \text { for all } s \neq t
\end{array}
$$

with $\delta_{i} \geq 0$ and $R_{s t}=\{0,1\}$. Equations 16 to 18 impose the requirement that estimated intersection demands are an element of the sufficient support set. See Hall and Huang (2001) for a discussion of alternative distance metrics.

I do not carry out inference based on the constrained estimator but direct the interested reader to Horowitz and Lee (2017). ${ }^{22}$ Under the assumption that the constraints are not binding in the population (i.e. violations of GARP arise from sampling variation), the constrained and unconstrained estimators have

\footnotetext{
${ }^{22}$ See also Freyberger and Reeves (2017).
} 
the same asymptotic distribution. However, in finite samples, the constrained and unconstrained estimators will be different, with different sampling distributions. When the rationality constraints are binding, the unconstrained estimator will be unlikely to sit at the interior of the constrained function space and the the asymptotic distribution of the constrained estimator will likely be non-standard (Blundell, Kristensen, and Matzkin 2014; Andrews 1999). Horowitz and Lee (2017) develop an approach for obtaining a uniform confidence band for $g_{t}^{k}(x)$ with a subset of binding, non-linear constraints that can be applied in the present context.

\subsection{Data}

I estimate mutually consistent demands using data from the U.K. Family Expenditure Survey. This is the exact same data used by Blundell, Browning, and Crawford (2008) and KS, allowing a straightforward comparison of the ease of implementing the respective methodologies. I make all the same assumptions as these papers when selecting and constructing the data. I use data between 1975 and 1999 for households with a car and at least one child. I consider choice over three composite commodities: food, service, and nondurable consumption goods. I assume that all consumers in a year face the same prices, and I use the same price data as these prior papers. ${ }^{23}$

Following KS, for my primary set of results, I estimate rational demands for blocks of seven consecutive periods by implementing the optimisation problem at Equation 12 to 18. Unlike KS, however, I follow the literature on e-bounds and estimate demands at intersection incomes rather than at median income in each period. ${ }^{24}$ I do this because, while some of the budget hyperplanes cross when median income is used, there are many more crossings when intersection incomes are used. This increases the probability that unconstrained demand predictions will fail GARP and that the rationality constraints under consideration in this paper will be binding.

I implement the optimisation problem for estimating mutually consistent intersection demands in MATLAB

\footnotetext{
${ }^{23}$ I would like to thank Yuichi Kitamura and Jorg Stoye for the high quality of their replication files, which made it very easy to construct the same data set as was used in Kitamura and Stoye (2017).

${ }^{24}$ Specifically, for the first budget in a block, $\widetilde{x}_{1}=\operatorname{median}(x)$. Then, for $t>1, \widetilde{x}_{t}=\mathbf{p}_{t}^{\prime} \widehat{\mathbf{q}}_{t-1}$ where $\widehat{\mathbf{q}}_{t-1}$ is the unconstrained demand prediction. When estimating intersection incomes as $\widetilde{x}_{t}=\mathbf{p}_{t}^{\prime} \widehat{\mathbf{q}}_{1}$ for $t>1$, it became infeasible to enumerate the set of rational choice types using the KS methodology but there was no change in the computation time of imposing the MILP constraints.
} 
on a standard desktop using the freely available IBM ILOG CPLEX optimisation solver. ${ }^{25}$ For implementing the mutual consistency constraints as defined in this paper, I do not make use of any parallel programming techniques as run times were always short (see below). To compare the performance of my formulation of the sufficient support set with an alternative based on KS, I use KS's replication code to enumerate the set of rational choice types making use of limited parrallelisation (4 cores).

A Gaussian kernel function and Silverman plug-in bandwidth are employed for the kernel weighting function. Many observations are associated with an income level 'far away' from the intersection incomes at which we predict demand, i.e. $K_{h}\left(x-x_{i}\right) / \sum_{j=1}^{N} K_{h}\left(x-x_{j}\right) \approx 0$. As these observations do not contribute to either the unconstrained or constrained kernel estimators, I do not include observations with a negligible kernel weighting in the optimisation procedure, i.e. if an observation $i$ has $K_{h}\left(x-x_{i}\right) / \sum_{j=1}^{N} K_{h}\left(x-x_{j}\right)<1 e^{-5}$ for all intersection demands. This is to avoid the number of variables in the optimisation procedure growing too large (the number of weight variables, $\delta_{i}$, rises linearly in the sample size as each observation is associated with its own weight).

\subsection{Results}

Table 1 summarises my empirical findings. It first displays whether unconstrained intersection demand predictions satisfy GARP. In only one of the eighteen blocks of periods do the unconstrained intersection demands pass GARP; in all other periods, demand predictions are not an element of the sufficient support set and need to be modified if they are to be used to generate e-bounds. This demonstrates the practical relevance of imposing joint rationality for state-of-the-art revealed preference techniques. $N$ shows the number of observations with a non-negligible kernel weighting in the unconstrained estimates which are re-weighted to ensure that demand predictions are an element of the sufficient support set.

I then report the number of constraints and variables associated with the MILP constraints defining the sufficient support sets. As I am predicting demands at 7 budgets of interest, there are 301 MILP constraints in each optimisation problem: 7 constraints ensure that the seven budget constraints bind; 42 constraints impose the direct revealed preference relation; 210 constraints impose transitivity of the revealed preference

\footnotetext{
${ }^{25}$ See the helpful guide produced by Laurens Cherchye, Bram De Rock, Thomas Demuynck and Frederic Vermeulen on the use of CPLEX for revealed preference analysis at: http://www.revealedpreferences.org/testingsimple.html
} 
relation; 42 constraints impose GARP on predictions. The number of variables in the optimisation procedure varies because of variation in the number of observations local to the intersection incomes in each problem. Taking the prediction problem for 1975-1981, there are $1789 \delta_{i}$ variables and 42 integer variables for imposing mutual consistency. There are 42 integer variables in all of the prediction problems.

An important metric is the time it takes to generate the necessary constraint matrices defining membership of the sufficient support set and to complete the optimisation procedure. In all cases except two, it took less than a second to build the mixed integer programming constraints and run the optimisation procedure to estimate the constrained demand predictions.

To demonstrate the utility of the MILP formulation, I also report the number of rational choice types associated with each prediction problem and the time taken to generate the constraint matrices defined in Kitamura and Stoye (2017). There are a large number of rational choice types associated with each prediction problem. The numbers are much larger than those reported in Kitamura and Stoye (2017) because here I am predicting demands at intersection incomes: this means that budget hyperplanes cross more often than in their original examples, resulting in many more rational choice types. Using four cores, the fastest run time for generating the matrices defining the rational choice types was 10 minutes; the longest run time was 25 minutes. This is substantially slower than generating the equivalent MILP formulation. 
Table 1: Empirical Results with 7 Periods

\begin{tabular}{c|cc|ccc|cc}
\hline \hline Years & GARP & $\mathrm{N}$ & \multicolumn{3}{|c}{ MILP Constraints } & \multicolumn{2}{c}{ KS Rational Type } \\
& & & \# Constraints & \# Variables & Time (s) & \# Rational Types & Time (s) \\
\hline $75-81$ & 0 & 1789 & 301 & 1831 & 0.9593 & $6,009,732$ & 884.8 \\
$76-82$ & 0 & 1717 & 301 & 1759 & 0.6590 & $8,169,999$ & 1350.4 \\
$77-83$ & 0 & 1644 & 301 & 1686 & 0.4808 & $5,314,901$ & 619.0 \\
$78-84$ & 0 & 1607 & 301 & 1649 & 0.5547 & $7,143,001$ & 1004.9 \\
$79-85$ & 0 & 1768 & 301 & 1810 & 0.6385 & $5,889,781$ & 790.4 \\
$80-86$ & 0 & 2155 & 301 & 2197 & 0.7977 & $5,923,070$ & 830.5 \\
$81-87$ & 1 & 2265 & 301 & 2307 & 0.8219 & $9,254,457$ & 1507.6 \\
$82-88$ & 0 & 2251 & 301 & 2293 & 0.9356 & $9,871,715$ & 1350.8 \\
$83-89$ & 0 & 2142 & 301 & 2184 & 0.8081 & $9,468,438$ & 1219.1 \\
$84-90$ & 0 & 2068 & 301 & 2110 & 1.2905 & $8,802,128$ & 1361.5 \\
$85-91$ & 0 & 2005 & 301 & 2047 & 1.1432 & $8,738,020$ & 955.2 \\
$86-92$ & 0 & 1860 & 301 & 1902 & 0.9416 & $8,561,194$ & 913.9 \\
$87-93$ & 0 & 1829 & 301 & 1871 & 0.6283 & $7,977,085$ & 911.0 \\
$88-94$ & 0 & 1785 & 301 & 1827 & 0.6092 & $8,269,301$ & 744.2 \\
$89-95$ & 0 & 1829 & 301 & 1871 & 0.5540 & $8,381,403$ & 665.1 \\
$90-96$ & 0 & 1848 & 301 & 1890 & 0.5938 & $8,955,417$ & 1076.8 \\
$91-97$ & 0 & 1830 & 301 & 1872 & 0.5826 & $7,330,365$ & 1023.3 \\
$92-98$ & 0 & 1854 & 301 & 1896 & 0.6337 & $7,721,041$ & 1154.9 \\
$93-99$ & 0 & 1791 & 301 & 1833 & 0.9417 & $9,952,538$ & 1522.7 \\
\hline \hline
\end{tabular}


Figure 7: Budget Hyperplanes \& Demand Predictions, 1975-1981

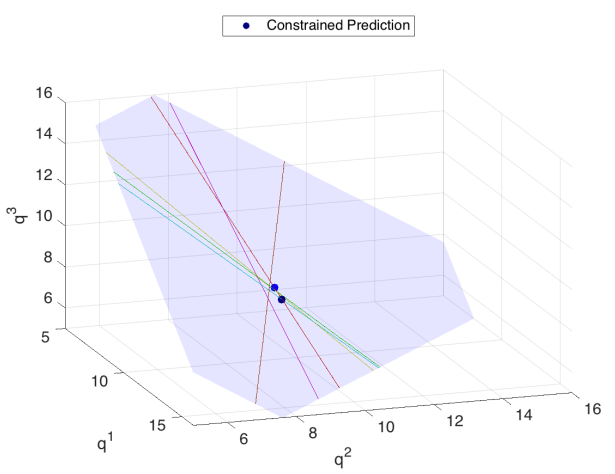

(a) 1975

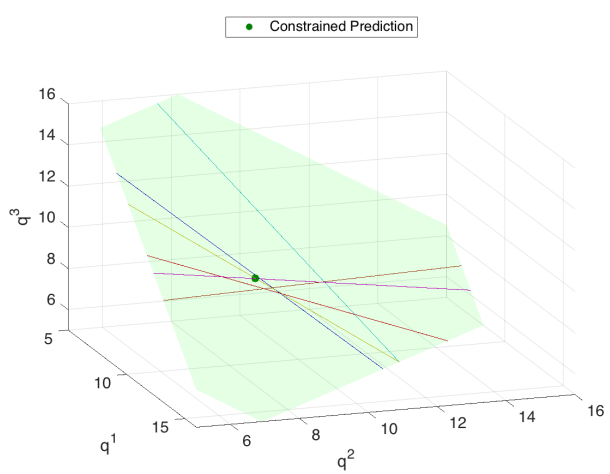

(c) 1977

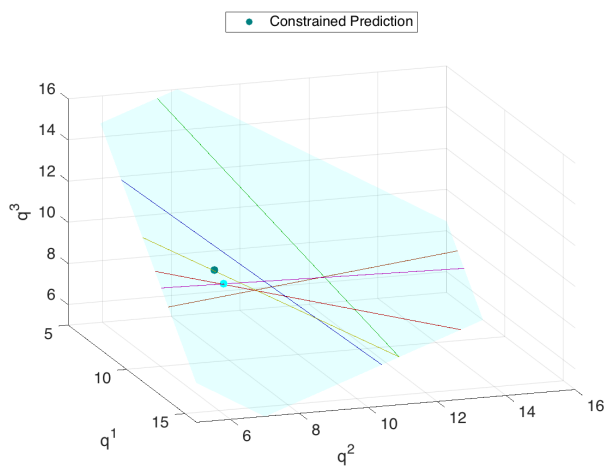

(e) 1979

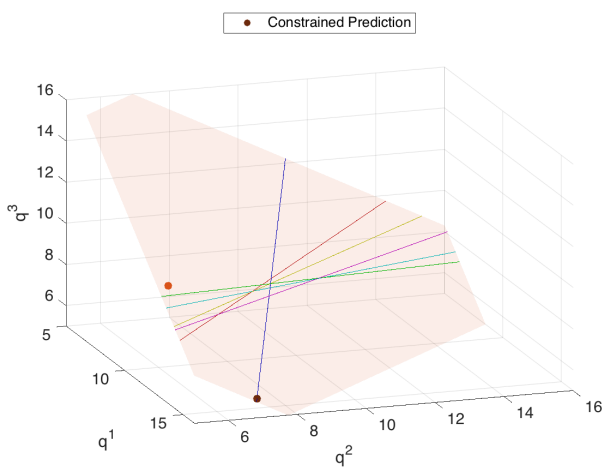

(g) 1981

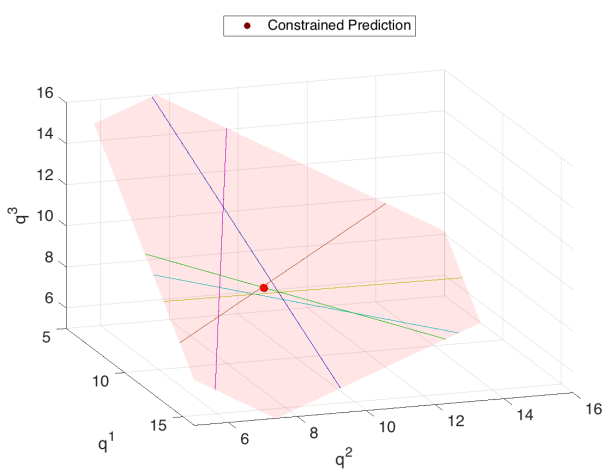

(b) 1976

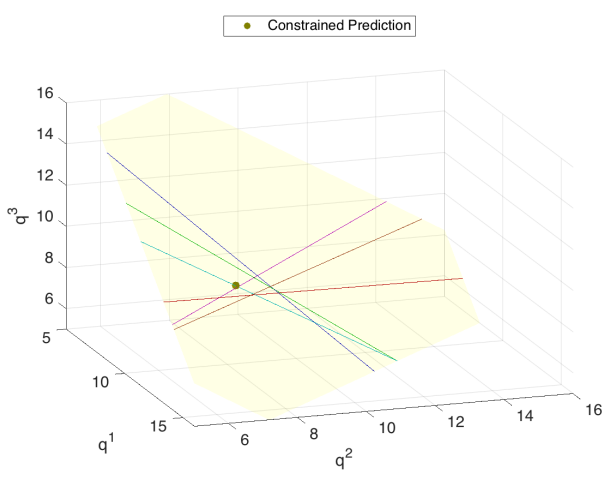

(d) 1978

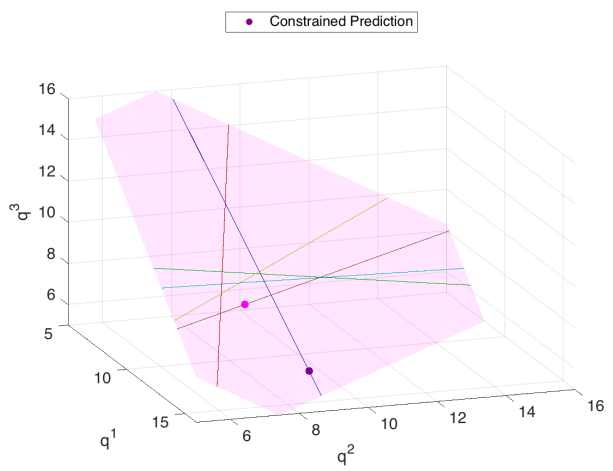

(f) 1980

Figure notes: each figure gives the budget hyperplane in that period (coloured area) with the unconstrained demand prediction (bright point) and the rationality constrained demand prediction(darker point). The lines correspond to the points of intersection with other budget hyperplanes. The colour of each intersection line indexes its year, e.g. the dark blue intersection lines correspond to the intersection with the 1975 budget hyperplane depicted in blue in panel (a). 
To provide insight into the nature of the demand predictions generated by the procedure, I focus on the solution output underlying the first prediction problem reported in Table 1 (1975-1981). ${ }^{26}$ Figure 7 shows the unconstrained prediction $\widehat{g}(\cdot)$ (bright point) and constrained demand prediction $\widehat{g}^{C}(\cdot)$ (darker dot) at each budget hyperplane (shaded) between 1975 and 1981. The points of intersection between budget hyperplanes are also given to illustrate the location of demand patches as in Kitamura and Stoye (2017).

The deviation between the constrained and unconstrained predictions is relatively small except for in the final two periods. ${ }^{27}$ To illustrate why demand in, for example, the final period must be modified for joint consistency of predictions, Figure 8 just shows a comparison of the unconstrained and constrained predictions in 1975 and 1981. In 1975, the unconstrained demand prediction is strictly affordable at the 1981 budget (the unconstrained prediction is on a patch 'under' the 1981 hyperplane). Similarly, the unconstrained demand prediction in 1981 is strictly affordable at the 1975 budget. Thus, these two demands violate revealed preference. Once the weight variables underlying the kernel prediction are appropriately modified by the MILP optimisation procedure, demand in 1975 is no longer strictly preferred to that at 1981 such that the constrained predictions satisfy GARP. Figure 9 gives the distribution of the constrained weight variables underlying the set of rational demand predictions for 1975-1981, $\widehat{\delta}$; in the unconstrained prediction, all weight variables equal one.

In order to establish how the properties of the procedure imposing the MILP constraints scale, I next report results for optimisation procedures with an increasing number of demand predictions. I again predict demands at intersection incomes ${ }^{28}$ sequentially adding each new period. I thus impose membership of the sufficient support set on between 2 and 25 demand predictions. Table 2 shows that both the number of variables and the number of constraints associated with the optimisation problem are increasing in the number of predictions. Once mutual consistency is imposed on 25 demand predictions simultaneously, the optimisation problem involves 15,025 constraints and 600 integer variables. ${ }^{29}$ However, the overall run time remains less than a minute demonstrating that the characterisation remains feasible to implement for practically sized problems.

\footnotetext{
${ }^{26}$ Please see Figure 11 in the Appendix for the distribution of rationality constrained weights in the otherprediction problems. ${ }^{27}$ Indeed, in 1976, 1977 and 1978 the differences are too small to be detectable on the graph.

${ }^{28}$ When the number of observations local to an intersection income falls below 100, I 're-set' the prediction income to the median income in that period.

$297228-6628=600$.
} 
Figure 8: Budget Hyperplanes \& Demand Predictions, 1975 and 1981

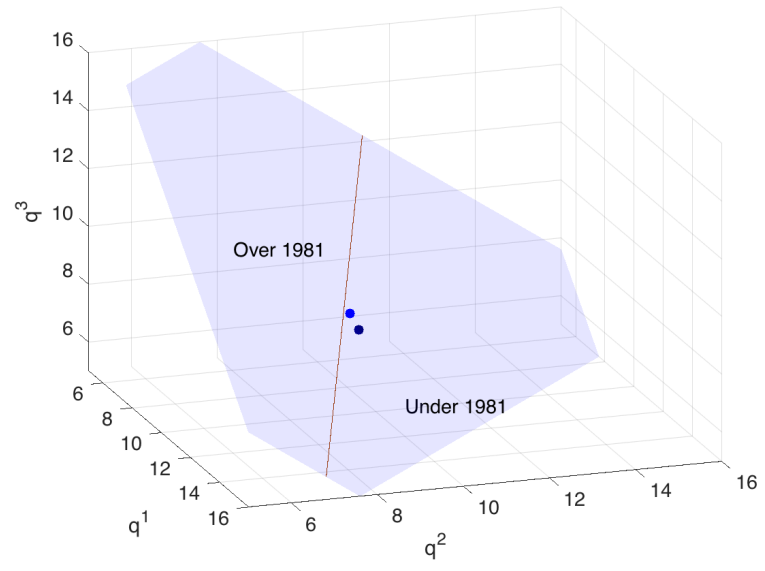

(a) 1975

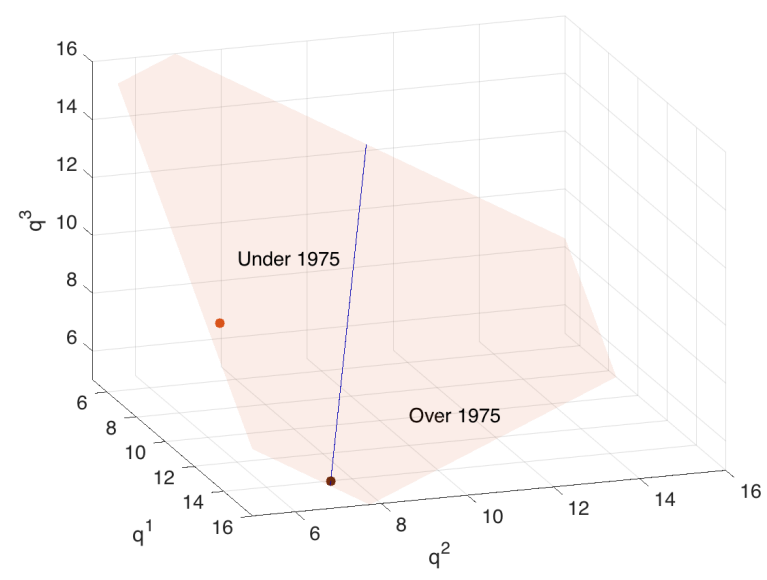

(b) 1981

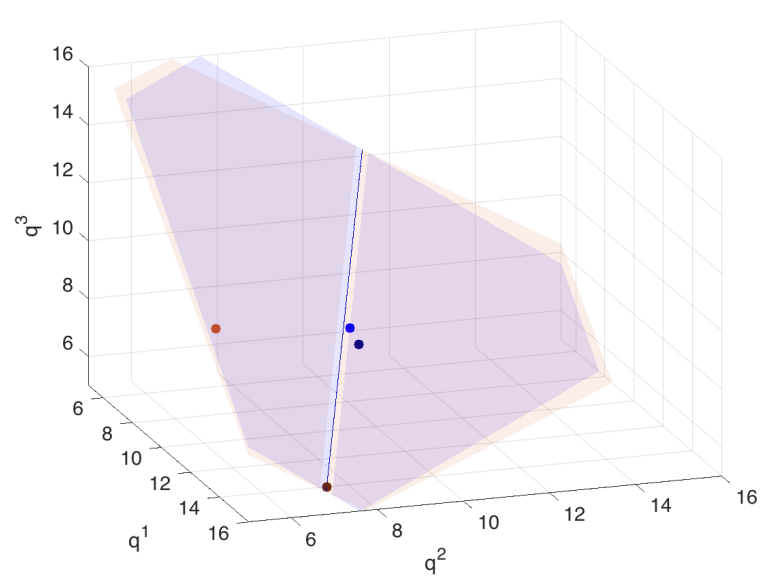

(c) Both

Figure notes: each figure gives the budget hyperplane in that period (coloured area) with the unconstrained demand prediction (bright point) and the rationality constrained demand prediction (darker point). The lines correspond to the points of intersection with other budget hyperplanes. The colour of each intersection line indexes its year, e.g. the dark blue intersection lines correspond to the intersection with the 1975 budget hyperplane depicted in blue in panel (a). The area labelled 'Over 1981' gives the patch on the 1975 budget hyperplane that cannot be afforded given the 1981 budget. Similarly the area labelled 'Under 1981 ' gives the patch on the 1975 budget hyperplane that is affordable given the 1981 budget. The areas 'Over 1975' and 'Under $1975^{\prime}$ are defined similarly. 
Figure 9: Histogram of Constrained Weights $\delta_{i t}, 1975-1981$

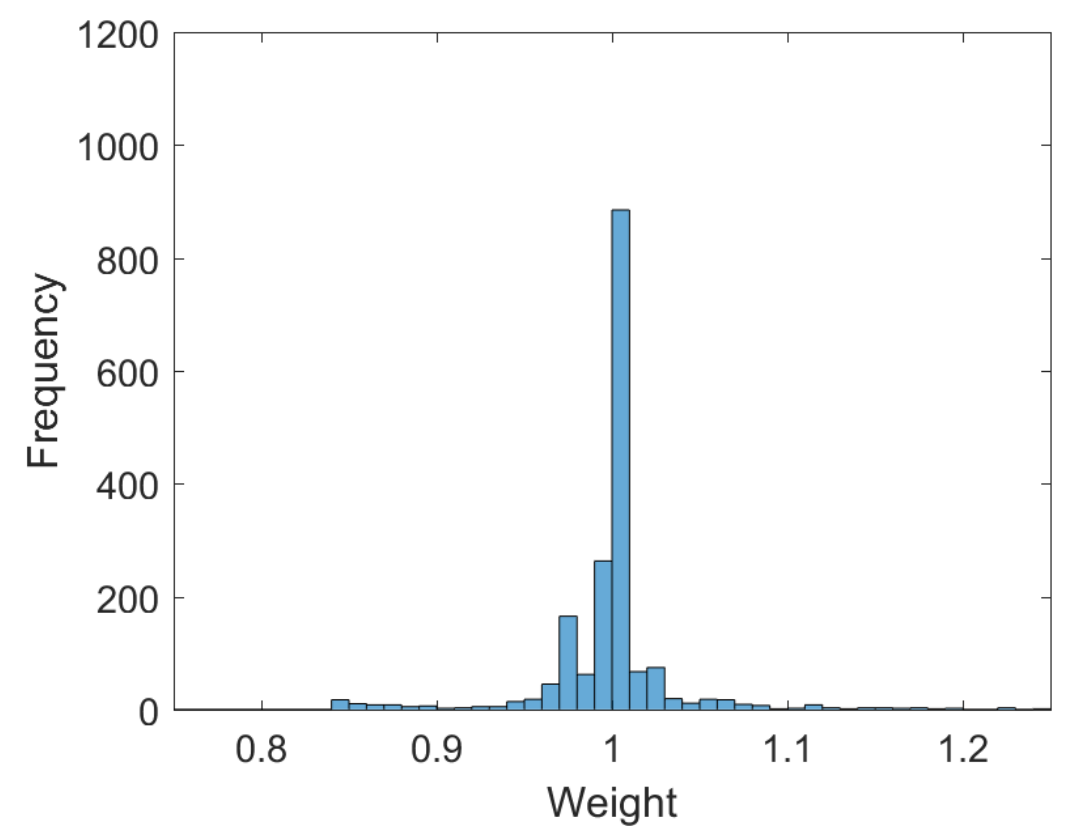

Figure notes: Histogram of rationality constrained weight variables underlying estimated intersection demands for the period 1975-1981.

Table 2: Empirical Results with Increasing Periods

\begin{tabular}{c|cc|ccc}
\hline \hline Years & $\mathrm{T}$ & $\mathrm{N}$ & \multicolumn{3}{|c}{ MILP Constraints } \\
& & & \# Constraints & \# Variables & Time (s) \\
\hline $75-76$ & 2 & 839 & 6 & 841 & 0.0780 \\
$75-77$ & 3 & 1216 & 21 & 1222 & 0.1418 \\
$75-78$ & 4 & 1493 & 52 & 1505 & 0.2511 \\
$75-79$ & 5 & 1684 & 105 & 1704 & 0.5031 \\
$75-80$ & 6 & 1751 & 186 & 1781 & 0.7014 \\
$75-81$ & 7 & 2185 & 301 & 2227 & 0.8911 \\
$75-82$ & 8 & 2599 & 456 & 2655 & 1.0121 \\
$75-83$ & 9 & 2963 & 657 & 3035 & 1.2222 \\
$75-84$ & 10 & 3272 & 910 & 3362 & 1.5885 \\
$75-85$ & 11 & 3542 & 1221 & 3652 & 2.9633 \\
$75-86$ & 12 & 3790 & 1596 & 3922 & 2.5283 \\
$75-87$ & 13 & 4016 & 2041 & 4172 & 7.4437 \\
$75-88$ & 14 & 4185 & 2562 & 4367 & 10.4117 \\
$75-89$ & 15 & 4318 & 3165 & 4528 & 13.2873 \\
$75-90$ & 16 & 4404 & 3856 & 4644 & 16.5469 \\
$75-91$ & 17 & 4683 & 4641 & 4955 & 15.3463 \\
$75-92$ & 18 & 4985 & 5526 & 5291 & 13.6930 \\
$75-93$ & 19 & 5241 & 6517 & 5583 & 15.5501 \\
$75-94$ & 20 & 5507 & 7620 & 5887 & 24.9242 \\
$75-95$ & 21 & 5778 & 8841 & 6198 & 40.9526 \\
$75-96$ & 22 & 6033 & 10186 & 6495 & 35.8285 \\
$75-97$ & 23 & 6234 & 11661 & 6740 & 31.6039 \\
$75-98$ & 24 & 6438 & 13272 & 6990 & 35.9405 \\
$75-99$ & 25 & 6628 & 15025 & 7228 & 59.1089 \\
\hline \hline
\end{tabular}




\section{Conclusion}

The revealed preference restrictions that are commonly imposed on demand predictions are not sufficient for rationality when predicting behaviour at a set of new budgets. When predicting over a set of intersecting budgets, not all combinations of demands from the Varian support sets will satisfy GARP. To ensure rationality of the combined set of predictions, mutual consistency must be imposed across predictions. The requirement of mutual consistency generates non-linearities in the typical revealed preference inequalities and can result in a non-convex set of demand predictions. This prevents standard linear programming methods from being employed to recover the support set.

This paper has provided a Mixed Integer Linear Programming representation of the mutual consistency constraints that can be applied with reasonable computational resources. Routes to enhance the efficiency of the procedure have been explored, giving possibilities for reducing the computational burden of the method. An empirical illustration using data from the UK Family Expenditure Survey served to demonstrate the implementation of the method to impose mutual consistency on nonparametric estimates of income expansion paths. This served to demonstrate the utility of the method for returning a rational set of 'intersection demands' from estimated income expansion paths, of the type required by Blundell, Browning and Crawford (2008) and Blundell, Kristensen and Matzkin (2014). The constraints were imposed with minimal computational resources on a standard desktop computer. Computation times of alternative procedures based on the enumeration of possible rational demands were an order of magnitude larger. Given the improvement of computation times achieved using the MILP representation, it would be interesting to explore the application of these constraints in the context of stochastic revealed preference to improve the applicability of Kitamura and Stoye (2017).

A full treatment of the asymptotic theory of the sufficient support set is beyond the scope of this paper. Depending on the application in mind, the results developed in Chernozhukov, Hong, and Tamer (2007) can be applied to develop an asymptotic theory of the mutually consistent bounds on demand responses. ${ }^{30}$ In practise, confidence bands will likely be constructed through subsampling as discussed in Chernozhukov, Hong, and Tamer (2007).

\footnotetext{
${ }^{30}$ See Blundell, Kristensen, and Matzkin (2014) for the application of the Chernozhukov, Hong, and Tamer (2007) machinery to develop confidence bounds on the Varian support set for quantile demands.
} 


\section{References}

Adams, A. (2014). Essays in Nonparametric Demand Analysis. University of Oxford Thesis.

Andreoni, J., B. Gillen, and W. Harbaugh (2011). The power of revealed preference tests: Ex-post evaluation of experimental design.

Andrews, D. W. (1999). Estimation when a parameter is on a boundary. Econometrica 67(6), 1341-1383.

Apesteguia, J. and M. A. Ballester (2015). A measure of rationality and welfare. Journal of Political Economy 123(6), 1278-1310.

Barseghyan, L., M. Coughlin, F. Molinari, and J. Teitelbaum (2018). Heterogeneous consideration sets and preferences.

Blundell, R. (2005). How revealing is revealed preference? Journal of the European Economic Association 3(2-3), 211-235.

Blundell, R., M. Browning, L. Cherchye, I. Crawford, B. De Rock, and F. Vermeulen (2015). Sharp for sarp: nonparametric bounds on counterfactual demands. American Economic Journal: Microeconomics 7(1), $43-60$.

Blundell, R., M. Browning, and I. Crawford (2003). Nonparametric engel curves and revealed preference. Econometrica 71(1), 205-240.

Blundell, R., M. Browning, and I. Crawford (2008). Best nonparametric bounds on demand responses. Econometrica 76(6), 1227-1262.

Blundell, R., J. Horowitz, and M. Parey (2012). Measuring the price responsiveness of gasoline demand: Economic shape restrictions and nonparametric demand estimation. Quantitative Economics 3(1), 2951.

Blundell, R., J. Horowitz, and M. Parey (2017). Nonparametric estimation of a nonseparable demand function under the slutsky inequality restriction. Review of Economics and Statistics.

Blundell, R., D. Kristensen, and R. Matzkin (2014). Bounding quantile demand functions using revealed preference inequalities. Journal of Econometrics 179(2), 112-127. 
Boyd, S. and L. Vandenberghe (2004). Convex optimization. Cambridge university press.

Cherchye, L., B. De Rock, J. Sabbe, and F. Vermeulen (2008). Nonparametric tests of collectively rational consumption behavior: an integer programming procedure. Journal of Econometrics 147(2), 258-265.

Chernozhukov, V., H. Hong, and E. Tamer (2007). Estimation and confidence regions for parameter sets in econometric models 1. Econometrica 75(5), 1243-1284.

Dean, M. and D. Martin (2016). Measuring rationality with the minimum cost of revealed preference violations. Review of Economics and Statistics 98(3), 524-534.

Echenique, F., S. Lee, and M. Shum (2011). The money pump as a measure of revealed preference violations. Journal of Political Economy 119(6), 1201-1223.

Freyberger, J. and B. Reeves (2017). Inference under shape restrictions. Available at SSRN 3011474.

Haag, B. R., S. Hoderlein, and K. Pendakur (2009). Testing and imposing slutsky symmetry in nonparametric demand systems. Journal of Econometrics 153(1), 33-50.

Hall, P. and L.-S. Huang (2001). Nonparametric kernel regression subject to monotonicity constraints. Annals of Statistics, 624-647.

Härdle, W. (1990). Applied nonparametric regression. Number 19. Cambridge university press.

Hoderlein, S. and J. Stoye (2015). Testing stochastic rationality and predicting stochastic demand: the case of two goods. Economic Theory Bulletin 3(2), 313-328.

Horowitz, J. and S. Lee (2017). Nonparametric estimation and inference under shape restrictions. Journal of Econometrics 201(1), 108-126.

Kitamura, Y. and J. Stoye (2017). Nonparametric analysis of random utility models. arXiv preprint $\operatorname{arXiv:1606.04819.}$

Kline, P. and M. Tartari (2016). Bounding the labor supply responses to a randomized welfare experiment: A revealed preference approach. American Economic Review 106(4), 972-1014.

Lima, R. M. and I. E. Grossmann (2011). Computational advances in solving mixed integer linear programming problems. 
Manski, C. F. (2014). Identification of income-leisure preferences and evaluation of income tax policy. Quantitative Economics 5(1), 145-174.

Mas-Colell, A. (1978). On revealed preference analysis. The Review of Economic Studies 45(1), 121-131.

Matzkin, R. L. (1994). Restrictions of economic theory in nonparametric methods. Handbook of econometrics 4, 2523-2558.

Nadaraya, E. A. (1964). On estimating regression. Theory of Probability \& Its Applications 9(1), 141-142.

Nobibon, F. T., L. Cherchye, Y. Crama, T. Demuynck, B. De Rock, and F. C. Spieksma (2016). Revealed preference tests of collectively rational consumption behavior: formulations and algorithms. Operations Research 64(6), 1197-1216.

Rose, H. (1958). Consistency of preference: the two-commodity case. The Review of Economic Studies 25(2), 124-125.

Varian, H. R. (1982). The nonparametric approach to demand analysis. Econometrica: Journal of the Econometric Society, 945-973.

Varian, H. R. (1990). Goodness-of-fit in optimizing models. Journal of Econometrics 46(1-2), 125-140.

Varian, H. R. (2012). Revealed preference and its applications. The Economic Journal 122(560), 332-338.

Watson, G. S. (1964). Smooth regression analysis. Sankhyā: The Indian Journal of Statistics, Series A, $359-372$.

\section{Appendix}

Proof of Proposition 1 Without loss of generality, let $\mathscr{P}=\{a, b\}$. First, define budget normalised prices, $\widetilde{\mathbf{p}}_{i}=\mathbf{p}_{i} / x_{i}$. Then define

$$
\begin{aligned}
& H_{-}(\widetilde{\mathbf{p}})=\left\{\mathbf{q}: \widetilde{\mathbf{p}}^{\prime} \mathbf{q} \leq 1\right\} \\
& H_{+}(\widetilde{\mathbf{p}})=\left\{\mathbf{q}: \widetilde{\mathbf{p}}^{\prime} \mathbf{q} \geq 1\right\}
\end{aligned}
$$

Since $S_{T}^{V}\left(\widetilde{\mathbf{p}}_{a}\right)$ and $S_{T}^{V}\left(\widetilde{\mathbf{p}}_{b}\right)$ intersect in the interior, $\exists \mathbf{q}_{a} \in H_{-}\left(\mathbf{p}_{b}\right) \cap S_{T}^{V}\left(\widetilde{\mathbf{p}}_{a}\right)$ and $\exists \mathbf{q}_{b} \in H_{-}\left(\mathbf{p}_{a}\right) \cap S_{T}^{V}\left(\widetilde{\mathbf{p}}_{b}\right)$, 
with $\mathbf{q}_{a} \neq \tilde{\mathbf{q}}$ and $\mathbf{q}_{b} \neq \tilde{\mathbf{q}}$. Define:

$$
\begin{aligned}
& \overline{\mathbf{q}}_{a} \in\left\{\mathbf{q}: \mathbf{q} \in S_{T}^{V}\left(\widetilde{\mathbf{p}}_{a}\right) \cap H_{+}\left(\mathbf{p}_{b}\right),\|\mathbf{q}-\tilde{\mathbf{q}}\|<\varepsilon\right\} \\
& \overline{\mathbf{q}}_{b} \in\left\{\mathbf{q}: \mathbf{q} \in S_{T}^{V}\left(\widetilde{\mathbf{p}}_{b}\right) \cap H_{+}\left(\mathbf{p}_{a}\right),\|\mathbf{q}-\tilde{\mathbf{q}}\|<\varepsilon\right\} .
\end{aligned}
$$

Then, taking the data set $D=\left\{\mathbf{p}_{a}, \mathbf{p}_{b},\left\{\mathbf{p}_{t}\right\}_{t \in \mathscr{D}} ; \mathbf{q}_{a}, \overline{\mathbf{q}}_{b},\left\{\mathbf{q}_{t}\right\}_{t \in \mathscr{D}}\right\}$, observe that:

- $D$ satisfies GARP.

From Kitamura and Stoye (2017), if there is a choice cycle of any finite length, then there is a cycle of length 2 or 3 - where a cycle of length 2 is a WARP violation. There are no cycles of length two - it is assumed that $\left\{\mathbf{p}_{t}, \mathbf{q}_{t}\right\}_{t \in \mathscr{D}}$ satisfies WARP, that $\left\{\mathbf{p}_{a},\left\{\mathbf{p}_{t}\right\}_{t \in \mathscr{D}} ; \mathbf{q}_{a},\left\{\mathbf{q}_{t}\right\}_{t \in \mathscr{D}}\right\}$ satisfies WARP by virtue of $\mathbf{q}_{a} \in S_{T}^{V}\left(\widetilde{\mathbf{p}}_{a}\right)$, and that $\left\{\mathbf{p}_{b},\left\{\mathbf{p}_{t}\right\}_{t \in \mathscr{D}} ; \overline{\mathbf{q}}_{b},\left\{\mathbf{q}_{t}\right\}_{t \in \mathscr{D}}\right\}$ satisfies WARP by virtue of $\mathbf{q}_{b} \in S_{T}^{V}\left(\widetilde{\mathbf{p}}_{b}\right)$. $\left\{\mathbf{p}_{a}, \mathbf{p}_{b} ; \mathbf{q}_{a}, \overline{\mathbf{q}}_{b}\right\}$ do not induce a WARP violation given that by construction:

$$
\begin{aligned}
& \mathbf{p}_{b}^{\prime} \mathbf{q}_{a}<1 \\
& \mathbf{p}_{a}^{\prime} \overline{\mathbf{q}}_{b}>1
\end{aligned}
$$

and $\neg\left(\mathbf{q}_{a} \mathbb{R} \overline{\mathbf{q}}_{b}\right)$.

There are no cycles of length three - Take $\mathbf{q}_{t} \in H_{-}\left(\widetilde{\mathbf{p}}_{a}\right) \cap H_{+}\left(\widetilde{\mathbf{p}}_{b}\right)$. We have: $\overline{\mathbf{q}}_{b} \mathbb{R}^{0} \mathbf{q}_{a} \mathbb{R}^{0} \mathbf{q}_{t}$. Thus, $\overline{\mathbf{q}}_{b} \mathbb{R} \mathbf{q} t$. This would induce an indirect revealed preference violation if $\mathbf{q}_{t} \mathbb{R} \overline{\mathbf{q}}_{b}$. However, for arbitrarily small $\epsilon, \overline{\mathbf{q}}_{b} \approx \tilde{\mathbf{q}}$ and $\tilde{\mathbf{q}} \mathbb{R} \mathbf{q}_{t}$.

- The indices $a$ and $b$ can be switched such that these arguments apply to $D^{\prime}=\left\{\mathbf{p}_{a}, \mathbf{p}_{b},\left\{\mathbf{p}_{t}\right\}_{t \in \mathscr{D}} ; \overline{\mathbf{q}}_{a}, \mathbf{q}_{b},\left\{\mathbf{q}_{t}\right\}_{t \in \mathscr{D}}\right\}$

Let an element of $S_{T}^{S}$, i.e. a single prediction, be given as the stacked column vector: $Q=\left[\mathbf{q}_{a}, \mathbf{q}_{b}\right]$. Let $Q^{a}=\left[\mathbf{q}_{a}, \overline{\mathbf{q}}_{b}\right]$ and $Q^{b}=\left[\overline{\mathbf{q}}_{a}, \mathbf{q}_{b}\right]$. From above, $Q^{a}, Q^{b} \in S^{S}$.Yet,

$$
\lambda Q^{a}+(1-\lambda) Q^{b} \notin S^{S}
$$


for some $\lambda \in(0,1)$. To see this note,

$$
\lambda Q^{a}+(1-\lambda) Q^{b}=\left[\begin{array}{c}
\lambda \mathbf{q}_{a}+(1-\lambda) \overline{\mathbf{q}}_{a} \\
\lambda \overline{\mathbf{q}}_{b}+(1-\lambda) \mathbf{q}_{b}
\end{array}\right],
$$

and that the choices $\left\{\lambda \mathbf{q}_{a}+(1-\lambda) \overline{\mathbf{q}}_{a}, \lambda \overline{\mathbf{q}}_{b}+(1-\lambda) \mathbf{q}_{b}\right\}$ violate WARP. It is the case that

$$
\begin{aligned}
& \mathbf{p}_{a}^{\prime} \mathbf{q}_{b}<1 \\
& \mathbf{p}_{a}^{\prime} \overline{\mathbf{q}}_{b} \approx 1
\end{aligned}
$$

Thus, for $\varepsilon$ sufficiently small, there exists a $\lambda$ such that

$$
\mathbf{p}_{a}^{\prime}\left(\lambda \overline{\mathbf{q}}_{b}+(1-\lambda) \mathbf{q}_{b}\right)<1
$$

Yet, by the same reasoning:

$$
\mathbf{p}_{b}^{\prime}\left(\lambda \overline{\mathbf{q}}_{a}+(1-\lambda) \mathbf{q}_{a}\right)<1
$$

leading to a revealed preference violation.

Proof of Proposition 2 Take some $\mathbf{q}_{a} \in S_{T}^{V}\left(\mathbf{p}_{a}, x_{a}\right)$. By definition, $\left\{\mathbf{q}_{a},\left\{\mathbf{q}_{t}\right\}_{t \in \mathscr{D}}\right\}$ satisfies GARP. There exists a utility function of the following structure that rationalises these choices (Varian, 1982):

$$
u(\mathbf{q})=\min _{i \in \mathscr{D} \cup a}\left\{u_{i}+\lambda_{i} \mathbf{p}_{i}^{\prime}\left(\mathbf{q}-\mathbf{q}_{i}\right)\right\}
$$

with $\lambda_{i}>0$. Let choices at $\forall b \in \mathscr{D} / a$ be given as:

$$
\mathbf{q}_{b}=\operatorname{argmax}_{\mathbf{q}}\left\{\min _{i \in \mathscr{D} \cup a}\left\{u_{i}+\lambda_{i} \mathbf{p}_{i}^{\prime}\left(\mathbf{q}-\mathbf{q}_{i}\right)\right\}\right\}
$$


subject to $\mathbf{p}_{b}^{\prime} \mathbf{q}=x_{b}$. By definition, this set of demand predictions satisfies GARP and each individual demand prediction is an element of its respective Varian support set.

Proof of Proposition 3. Constraints [1] and [2] flow directly from [S1] and [S2]. Constraints [3], [4], [5] impose GARP, and thus [S3] on the set of demands at $\mathscr{B}$.

Constraint [3] imposes the requirement that $\mathbf{q}_{b} \mathbb{R} \mathbf{q}_{a}$, i.e. $R_{b a}=1$, if $\mathbf{p}_{b}^{\prime} \mathbf{q}_{a} \leq x_{b}$, thereby defining the direct revealed preference relation. To see this note that if $R_{b a}=0$, Constraint [3] evaluates as $\mathbf{p}_{b}^{\prime} \mathbf{q}_{a}>x_{b}$, which is a contradiction. If $\mathbf{p}_{b}^{\prime} \mathbf{q}_{a}>x_{b}, R_{b a}=1$ or $R_{b a}=0$ are permissible.

Constraint [4] imposes transitivity of the revealed preference relation. If $\mathbf{q}_{a} \mathbb{R}^{0} \mathbf{q}_{b}$ and $\mathbf{q}_{b} \mathbb{R}^{0} \mathbf{q}_{c}$, then we require $\mathbf{q}_{a} \mathbb{R} \mathbf{q}_{c}$. If $R_{a b}=1$ and $R_{b c}=1$, Constraint [4] is violated unless $R_{a c}=1$. The integer variables $R_{i j}$ are thus equivalent to the revealed preference relation.

Constraint [5] imposes GARP. If $R_{a b}=1$, then $\mathbf{p}_{b}^{\prime} \mathbf{q}_{a} \geq 1$, or the constraint is violated. If $R_{a b}=0$, $\mathbf{p}_{b}^{\prime} \mathbf{q}_{a} \lessgtr 1$

Proof of Proposition 4 The proof of Proposition 4 follows from the proof of Theorem 3.2 in Kitamura and Stoye (2017). First, define budget normalised prices, $\widetilde{\mathbf{p}}_{i}=\mathbf{p}_{i} / x_{i}$.

For $\forall a \in \mathscr{P}_{m}$ and $\forall b \in \mathscr{P}_{n}$ with $m \neq n$, either $\widetilde{\mathbf{p}}_{a}^{\prime} \mathbf{q}<1$ for all $\mathbf{q}>0$ such that $\widetilde{\mathbf{p}}_{b} \mathbf{q} \leq 1$ or $\widetilde{\mathbf{p}}_{b}^{\prime} \mathbf{q}<1$ for all $\mathbf{q}>0$ such that $\widetilde{\mathbf{p}}_{a} \mathbf{q} \leq 1$. Let the former hold without loss of generality.

By construction, $\mathbf{q}_{i} \in S_{T}^{V}\left(\widetilde{\mathbf{p}}_{i}\right)$ for all $i \in \mathscr{D}$. Choices at $\widetilde{\mathbf{p}}_{a}, a \in \mathscr{P}_{m}$, and at $\widetilde{\mathbf{p}}_{b}, b \in \mathscr{P}_{n}$, cannot generate a length two cycle because $\widetilde{\mathbf{p}}_{a}^{\prime} \mathbf{q}_{b} \leq 1$ for all $\mathbf{q}_{b} \in S_{T}^{V}\left(\widetilde{\mathbf{p}}_{b}\right)$ and $\widetilde{\mathbf{p}}_{b}^{\prime} \mathbf{q}_{a} \geq 1$ for all $\mathbf{q}_{a} \in S_{T}^{V}\left(\widetilde{\mathbf{p}}_{a}\right)$, with equality only possible if at a corner. No length three cycles can be generated. By construction, $\mathbf{q}_{a} \mathbb{R}^{0} \mathbf{q}_{b}$ for all $\mathbf{q}_{a} \in S_{T}^{V}\left(\widetilde{\mathbf{p}}_{a}\right)$ and $\mathbf{q}_{b} \in S_{T}^{V}\left(\widetilde{\mathbf{p}}_{b}\right)$. For any $\mathbf{q}_{t}$ such that $\mathbf{q}_{b} \mathbb{R}^{0} \mathbf{q}_{t}$, the $\mathbf{q}_{a} \mathbb{R}^{0} \mathbf{q}_{t}$ as $\widetilde{\mathbf{p}}_{a}^{\prime} \mathbf{q}<1$ for all $\mathbf{q}>0$ such that $\widetilde{\mathbf{p}}_{b} \mathbf{q} \leq 1$. For any $\mathbf{q}_{t}$ such that $\mathbf{q}_{t} \mathbb{R}^{0} \mathbf{q}_{a}, \mathbf{q}_{t} \mathbb{R}^{0} \mathbf{q}_{b}$.

\subsection{Formal Treatment of the Connection to KS}

KS characterise their approach in terms of 'patches' and 'choice types'. Patches form the coarsest partition of budget sets such that no budget set intersects the interior of an another at the interior of a patch. Rational choice types are defined as a combination of 'patches' across budgets of interest. The sufficient support set 
Figure 10: Multiple Predictions

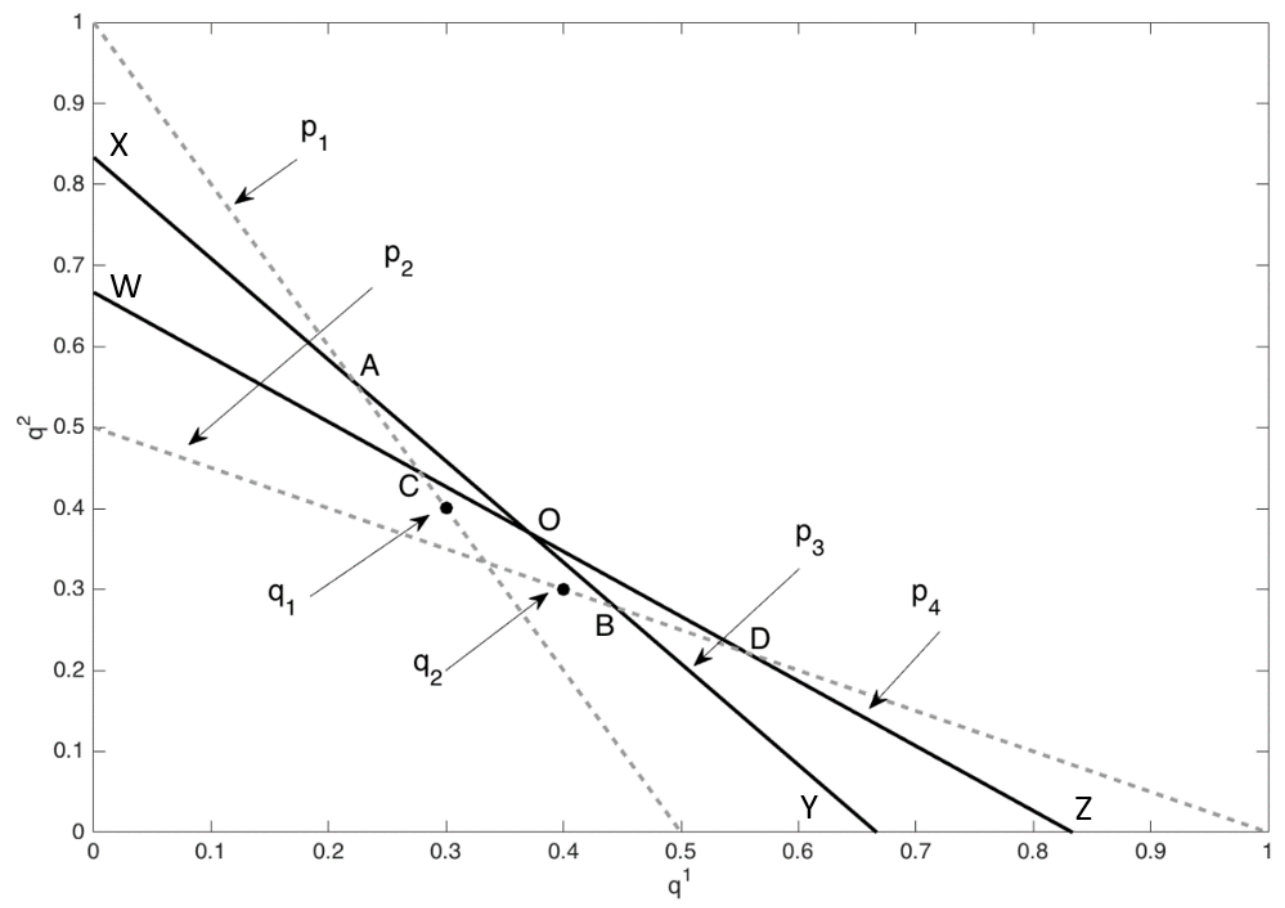

can be defined as a finite list of rational 'choice types' as well as by the MILP characterisation put forward in this paper.

To use the language of Kitamura and Stoye (2017), in our illustrative prediction problem, there are four 'patches' of interest on the budgets $\left\{\mathbf{p}_{3}, x_{3}\right\}$ and $\left\{\mathbf{p}_{4}, x_{4}\right\}$. For example, on $\left\{\mathbf{p}_{3}, x_{3}\right\}$, the first patch consistent with past demands is given by $\mathrm{AO}$ and the second by $\mathrm{OB}$.

Following Kitamura and Stoye (2017), let $A$ encode the rational combination of patches corresponding to the choice problem in Figure 10. Each column of $A$ encodes the demand behaviour of a given rational choice type. Each column of $A$ thus also corresponds to a feasible combination of binary variables in our integer programming formulation. Applying this to our illustrative example, the first column of $A$ corresponds to a consumer demanding $\mathbf{q}_{3} \in A O$ and $\mathbf{q}_{4} \in O D$. This corresponds to $R_{34}=1$ and $R_{43}=1$ in Proposition 3 . Each row of $A$ corresponds to a particular patch: 


$$
A=\begin{aligned}
& X A \\
& A O \\
& C O \\
& C O \\
& O D \\
& D Z
\end{aligned}\left[\begin{array}{lll}
0 & 0 & 0 \\
1 & 1 & 0 \\
0 & 0 & 1 \\
0 & 0 & 0 \\
0 & 0 & 0 \\
0 & 1 & 0 \\
1 & 0 & 1 \\
0 & 0 & 0
\end{array}\right]
$$

Why then is the MIP representation of the mutual consistency constraints novel and useful? Couldn't one simply restrict demand combinations to those consistent with a single column of $A$ ? The most straightforward way that I could think of translating KS's framework into constraints amenable to this problem is as follows:

$$
\begin{aligned}
\mathbf{q}_{a} \geq M(A \circ \bar{Q}) \boldsymbol{\pi} & \text { for } a=T+1, \ldots, B \\
\mathbf{q}_{a} \leq M(A \circ \underline{Q}) \boldsymbol{\pi} & \text { for } a=T+1, \ldots, B \\
1 & =\mathbb{1}^{\prime} \boldsymbol{\pi} \\
\pi_{i} & =\{0,1\}
\end{aligned}
$$

where $\bar{Q}$ and $\underline{Q}$ are $K \times \operatorname{columns}(A)$ matrices for which $\bar{Q}_{k i}$ and $\underline{Q}_{k i}$ give the lower and upper bounds on the quantity of good $k$ associated with each patch and choice type. $M$ is an $|\mathscr{P}| \times \operatorname{rows}(A)$ matrix with $M_{i j}=1$ if patch $j$ is on budget $T+i . \pi$ is a columns $(A) \times 1$ vector of binary indicators that indicate which rational choice type (column of A) is imposed. o represents the Hadamard product. As discussed in Section 4.2 and 5 , when there are a large number of rational types because many budget hyperplanes cross, constructing the $A$ matrix is a very computationally burdensome procedure.

\subsection{Additional Histograms of Weight Variables}


Figure 11: Histrograms of Constrained Kernel Weights

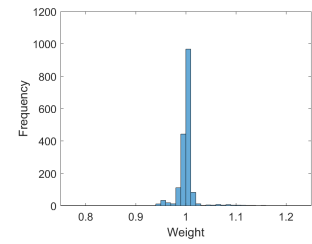

1976-1982

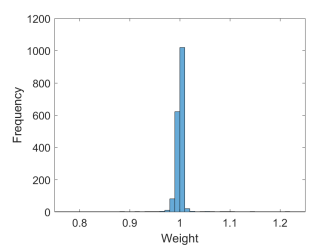

1979-1985

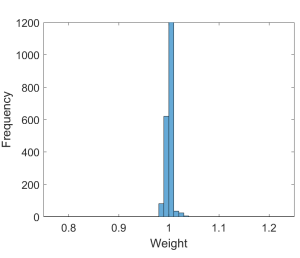

1982-1988

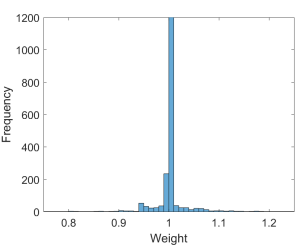

$1985-1991$

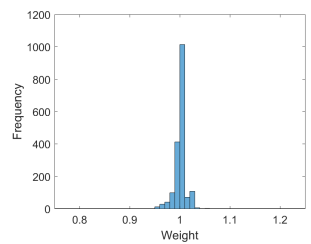

1988-1994

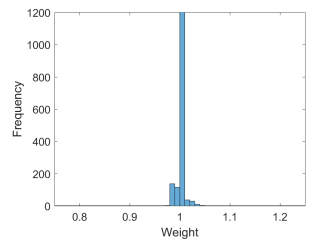

1991-1997

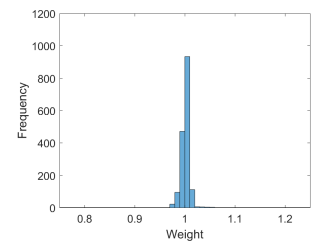

1977-1983

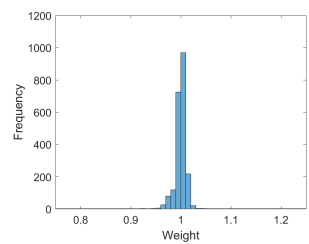

$1980-1986$

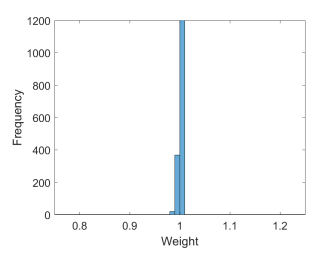

$1983-1989$

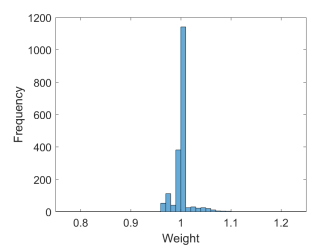

1986-1992

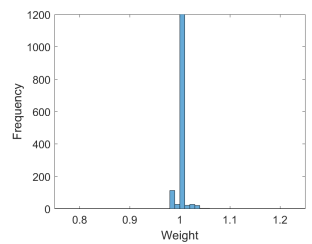

1989-1995

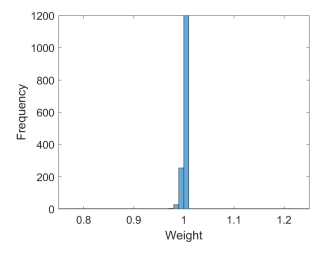

1992-1998

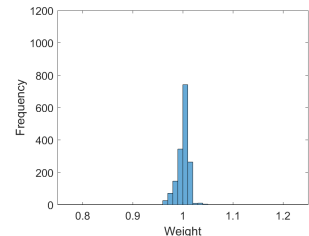

1978-1984

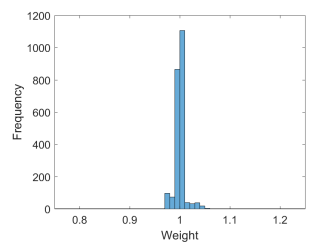

$1981-1987$

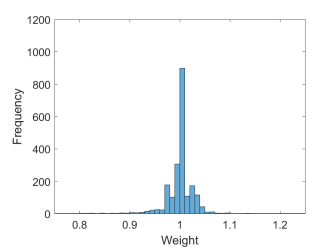

1984-1990

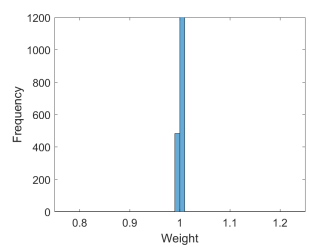

1987-1993

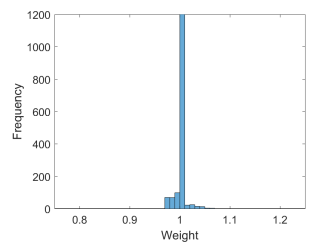

1990-1996

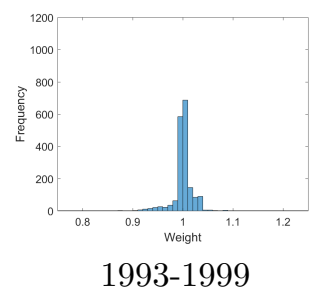

Figure notes: Histogram of rationality constrained weight variables underlying estimated intersection demands for each prediction problem. 